\title{
FOURIER INTEGRAL OPERATORS WITH OPEN UMBRELLAS AND SEISMIC INVERSION FOR CUSP CAUSTICS
}

\author{
Raluca Felea and Allan Greenleaf
}

\begin{abstract}
The composition of Fourier integral operators (FIOs) need not in general be an FIO. Motivated by the problem of linearized seismic inversion in the presence of cusp caustics for the background sound speed, we consider FIOs whose canonical relations have certain two-sided cusp degeneracies, and show that the resulting compositions have wave-front relations in the union of the diagonal and an open umbrella, the simplest type of singular Lagrangian manifold.
\end{abstract}

\section{Introduction}

A fundamental problem concerning Fourier integral operators (FIOs) is that, outside of the standard transverse [19] and clean intersection [5, 32] calculus, the composition of two FIOs is typically not another FIO. Describing the operators resulting from the composition of completely general FIOs and placing them in a usable class, with a symbol calculus, Sobolev space estimates, and the possibility of constructing parametrices under suitable ellipticity conditions, is at this point a distant prospect. Some progress has been made for specific geometries arising in integral geometry [16] and inverse problems $[6,7,21,28]$. In all of these works, the compositions were shown to belong to an existing class, the pseudodifferential operators with singular symbols, which are not FIOs and were introduced to construct parametrices for operators of real or complex principal type $[24,18,26]$. Such operators have wave front relation, i.e., the wave front set of the Schwartz kernel, contained in the union of the diagonal and another smooth canonical relation which intersects the diagonal cleanly.

In the present paper, motivated by a linearized inverse problem from seismology for the acoustic wave equation, we analyze FIOs having a special type of degeneracy, which we call a flat two-sided cusp, and show that their composition results in operators which are fundamentally more singular than those in the references above.

FIOs with two-sided cusps, i.e., those for which the projections from the canonical relation both to the left and the right are cusps, arise naturally when considering generalized Radon transforms. They were previously studied in terms of $L^{2}$ Sobolev regularity properties; there is a loss of $1 / 4$ derivative compared with the nondegenerate case of local canonical graphs $[3,13]$. Lebesgue space estimates for FIOs with onesided cusps have also been obtained, with the operator $T^{*} T$ making an appearance through Strichartz estimate type arguments [12]; however, a composition calculus has not been studied in its own right. In this paper, we analyze compositions such as $T^{*} T$ for operators associated with what we call flat two-sided cusps (defined in Sec. 2.5). Our main motivation for studying these comes from the following theorem; background on the seismology problem can be found in Sec. 3 . 
Theorem 1.1. If $c_{0}(x)$ is a smooth background sound speed whose rays, emanating from a source $s_{0}$, have caustics of cusp type, then the resulting single-source linearized scattering operator $F$ is an FIO of order 1 associated with a flat two-sided cusp, $C$.

In the absence of caustics, Beylkin [2] showed that $F$ is associated with a local canonical graph, so that the normal operator $F^{*} F$ is a pseudodifferential operator. In the presence of caustics of fold type, $C$ is a two-sided fold [28], and $W F\left(F^{*} F\right)$ is contained in the union of the diagonal $\Delta$ and a smooth canonical relation $\widetilde{C}$, resulting in a strong, nonremovable imaging artifact; more can be said, with $F^{*} F$ belonging $[28,6,7,8]$ to a class of previously introduced operators $[24,18,26]$. Here, we show that for cusp caustics, $W F\left(F^{*} F\right)$ is contained in a union $\Delta \cup \widetilde{C}$, but with $\widetilde{C}$ no longer smooth, as in the case of fold caustics; rather, $\widetilde{C}$ has the structure of the simplest kind of singular Lagrangian manifold, an open umbrella [9] (see Sec. 2.3 for definition).

Theorem 1.2. If $X, Y$ are three-dimensional manifolds, $C \subset T^{*} X \times T^{*} Y$ is a flat two-sided cusp canonical relation, and $A, B: \mathcal{E}^{\prime}(Y) \longrightarrow \mathcal{E}^{\prime}(X)$ are properly supported FIOs associated to $C$ of orders $m, m^{\prime} \in \mathbb{R}$, resp., then $W F\left(B^{*} A\right) \subset \Delta \cup \widetilde{C}$, where $\Delta$ is the diagonal of $T^{*} Y \times T^{*} Y$ and $\widetilde{C}$ is an open umbrella intersecting $\Delta$ in codimension one. Microlocally away from $\Delta \cap \widetilde{C}, B^{*} A$ is of order $m+m^{\prime}$ on both $\Delta$ and $\widetilde{C}$.

As applied to the seismic imaging problem, the results here have negative implications (as do the results of $[28,6]$ in the case of fold caustics): even microlocally away from $\Delta \cap \widetilde{C}$, the non-pseudodifferential part of the normal operator has the same order as the pseudodifferential part, resulting in a strong, nonremovable artifact. However, our work provides a precise description of the imaging artifacts resulting from cusp caustics, observed by Nolan and Symes [29] in a model 2D case. We also note that operators with wave front relation in the union of $\Delta$ and a canonical relation having a conical singularity were considered by Melrose and Uhlmann [25] in another setting.

We begin in Sec. 2 with a simple example from harmonic analysis of a FIO having two-sided cusps, recall some basic singularity theory, and examine the corresponding normal operator. In Sec. 2.5 we define the general class of canonical relations having this structure, the flat two-sided cusps. In Sec. 3, we describe the linearized inverse problem from seismology, and prove Thm. 1.1. In Sec. 4 we derive a weak normal form for general flat two-sided cusps, very close to the model examined in Sec. 2. Finally, in Sec. 5, we use the oscillatory representations found in Sec. 4 to analyze the composition $B^{*} A$ for two FIOs associated with such a canonical relation, proving Thm. 1.2. We point out that the technique of deriving weak normal forms for degenerate FIOs and using them to prove composition theorems has its origins in [16] and was continued in $[21,7]$; different notions of weak normal forms were used to obtain estimates in $[11,12]$.

\section{Example of an FIO with a flat two-sided cusp}

2.1. A generalized Radon transform. We start with a simple example of a generalized Radon transform that incorporates the features of the linearized seismic inversion problem in which we are ultimately interested. In fact, we will later show that perturbations of this example serve as weak normal forms for the general class of operators with flat two-sided cusps. 
The operator averaging over translates in $\mathbb{R}^{4}$ of a curve $\gamma(t)$ such that $\dot{\gamma}, \ddot{\gamma}, \dddot{\gamma}, \gamma^{(4)}$ are linearly independent is an FIO having a canonical relation which is a two-sided cusp [12], but the same holds for the curve $\gamma(t)=\left(t, t^{2}, t^{4}\right)$ in $\mathbb{R}^{3}[13,14]$, and this is the model we will use.

Thus, consider the generalized Radon transform $R_{0}: \mathcal{D}^{\prime}\left(\mathbb{R}^{3}\right) \longrightarrow \mathcal{D}^{\prime}\left(\mathbb{R}^{3}\right)$,

$$
\begin{aligned}
& R_{0} f(x)=\int f\left(x-\left(t, t^{2}, t^{4}\right)\right) \chi(t) d t \\
= & \int_{\mathbb{R}^{3}} e^{i\left(\left(x_{2}-y_{2}-\left(x_{1}-y_{1}\right)^{2}\right) \theta_{2}+\left(x_{3}-y_{3}-\left(x_{1}-y_{1}\right)^{4}\right) \theta_{3}\right)} f(y) \chi\left(x_{1}-y_{1}\right) 1(\theta) d \theta_{2} d \theta_{3} d y,
\end{aligned}
$$

where $\chi \in C_{0}^{\infty}(\mathbb{R})$ is a fixed cutoff function. The associated canonical relation, the conormal bundle $C_{0}=N^{*} Z^{\prime} \subset\left(T^{*} \mathbb{R}^{3} \backslash 0\right) \times\left(T^{*} \mathbb{R}^{3} \backslash 0\right)$, where $Z=\operatorname{supp}\left(K_{R_{0}}\right) \subset$ $\mathbb{R}^{3} \times \mathbb{R}^{3}$ and $(x, \xi ; y, \eta)^{\prime}=(x, \xi ; y,-\eta)$ is standard notation for the twist map, is

$$
\begin{gathered}
C_{0}=\left\{\left(x_{1}, x_{2}, x_{3},-2\left(x_{1}-y_{1}\right) \theta_{2}-4\left(x_{1}-y_{1}\right)^{3} \theta_{3}, \theta_{2}, \theta_{3} ;\right.\right. \\
\left.y_{1}, y_{2}, y_{3},-2\left(x_{1}-y_{1}\right) \theta_{2}-4\left(x_{1}-y_{1}\right)^{3} \theta_{3}, \theta_{2}, \theta_{3}\right): \\
\left.x_{2}-y_{2}-\left(x_{1}-y_{1}\right)^{2}=x_{3}-y_{3}-\left(x_{1}-y_{1}\right)^{4}=0, \theta \neq 0\right\} .
\end{gathered}
$$

Now briefly recall:

Definition 2.1. (a) A submanifold $\Gamma \subset T^{*} \mathbb{R}^{n}$ is coisotropic (or involutive) if $\Gamma$ can microlocally be written as $\left\{p_{1}=\cdots=p_{k}=0\right\}$, with all Poisson brackets $\left\{p_{i}, p_{j}\right\}=0$. (b) $\Gamma$ is nonradial if, $\forall(x, \xi) \in \Gamma$, the radial vector field $\xi \cdot \partial_{\xi} \notin T \Gamma^{\omega}$, the annihilator of $T \Gamma$ w.r.t the symplectic form $\omega_{T^{*} \mathbb{R}^{n}}$.

(It follows from the homogeneous Darboux theorem [19] that a codimension $k$ nonradial coisotropic can be mapped to $\left\{\xi_{1}=\cdots=\xi_{k}=0\right\}$ by a homogeneous canonical transformation.)

We will show that $C_{0}$ has several properties, which remarkably also hold in the totally unrelated seismic imaging problem: (i) the projections both to the left and right, $\pi_{L}, \pi_{R}: C_{0} \longrightarrow T^{*} \mathbb{R}^{3} \backslash 0$, have (Whitney) cusp degeneracies; (ii) the cusp points for the two projections are the same, $\Sigma_{1,1}\left(\pi_{L}\right)=\Sigma_{1,1}\left(\pi_{R}\right):=\Sigma_{1,1}$; and (iii) the images of the cusp points, $\pi_{L}\left(\Sigma_{1,1}\right)$ and $\pi_{R}\left(\Sigma_{1,1}\right)$, are coisotropic, nonradial submanifolds of $T^{*} \mathbb{R}^{3}$. Finally, we note that conditions (ii) and (iii) are unstable and quite special among canonical relations satisfying (i); see the Remarks in Sec. 2.5 below.

2.2. Singularity classes. We first recall some basic facts about cusps and refer to $[33,27,10]$ for more details. Let $f: \mathbb{R}^{N} \rightarrow \mathbb{R}^{N}$ be a smooth function. We say that $f$ drops rank simply at $p$ if rank $(d f)_{p}=N-1$ and if $(d(\operatorname{det} d f))_{p} \neq 0$, so that $\Sigma_{1}(f):=\left\{x \in \mathbb{R}^{N}: \operatorname{det}(d f(x))=0\right\}$, the corank one critical set of $f$, is a smooth hypersurface near $p$. If ker $d f_{p} \not \subset T_{p} \Sigma_{1}(f)$, then $f$ has a fold singularity, and $\left.f\right|_{\Sigma_{1}(f)}$ is an immersion. Considering the more degenerate case when ker $d f_{p} \subset T_{p} \Sigma_{1}(f)$, one may choose a nonzero vector field $v$ along $\Sigma_{1}(f)$ such that $v \in \operatorname{ker} d f_{p}$, so that $v$ is tangent to $\Sigma_{1}(f)$ at $p$. Let $g$ be a smooth function such that $\left.g\right|_{\Sigma_{1}(f)}=0$ and $d g_{p} \neq 0$, e.g., $g=\operatorname{det} d f$. Thus, $d g(v)$ has a zero at $p$.

Definition 2.2. $f$ has a (Whitney) cusp at $\mathrm{p}$ if $d g(v)$ has a simple zero at $p$. 
$\Sigma_{1,1}(f)$, the cusp set of $f$, is then codimension 2 , and $\left.f\right|_{\Sigma_{1,1}(f)}$ is an immersion. One may use adapted coordinates to clarify this. These are local coordinates such that $f\left(x_{1}, x_{2}, \ldots, x_{N}\right)=\left(x_{1}, x_{2}, \ldots, x_{N-1}, h(x)\right)$ and $h(0)=0$ [27]. Then, $\Sigma_{1}(f)=$ $\left\{x: \frac{\partial h}{\partial x_{N}}(0)=0\right\}$, and $f$ has a cusp singularity at 0 iff $\frac{\partial^{2} h}{\partial x_{N}^{2}}(0)=0, \quad \frac{\partial^{3} h}{\partial x_{N}^{3}}(0) \neq 0$ and $\operatorname{rank}\left[d_{x}\left(\frac{\partial h}{\partial x_{N}}\right), d_{x}\left(\frac{\partial^{2} h}{\partial x_{N}^{2}}\right)\right]=2$. These conditions are adapted coordinate-independent, and the notion of a cusp makes sense for any smooth mapping between $N$-dimensional manifolds. In suitable smooth coordinates on the domain and range spaces, any map with a cusp singularity can be put into the local normal form, $f\left(x_{1}, x_{2}, \ldots x_{N}\right)=$ $\left(x_{1}, x_{2}, \ldots, x_{N-1}, x_{N-1} x_{N}+x_{N}^{3}\right)$.

For the canonical relation $C_{0}$, the projection to the left, $\pi_{L}: C_{0} \longrightarrow T^{*} \mathbb{R}^{3}$, is $\pi_{L}\left(x_{1}, x_{2}, x_{3}, y_{1}, \theta_{2}, \theta_{3}\right)=\left(x_{1}, x_{2}, x_{3},-2\left(x_{1}-y_{1}\right) \theta_{2}-4\left(x_{1}-y_{1}\right)^{3} \theta_{3}, \theta_{2}, \theta_{3}\right) ;$ hence, letting $\alpha=2 \theta_{2}+12\left(x_{1}-y_{1}\right)^{2} \theta_{3}, \beta=-2\left(x_{1}-y_{1}\right)$ and $\gamma=-4\left(x_{1}-y_{1}\right)^{3}$, one has

$$
d \pi_{L}=\left[\begin{array}{cccccc}
1 & 0 & 0 & 0 & 0 & 0 \\
0 & 1 & 0 & 0 & 0 & 0 \\
0 & 0 & 1 & 0 & 0 & 0 \\
-\alpha & 0 & 0 & \alpha & \beta & \gamma \\
0 & 0 & 0 & 0 & 1 & 0 \\
0 & 0 & 0 & 0 & 0 & 1
\end{array}\right]
$$

which has $\operatorname{det} d \pi_{L}=2 \theta_{2}+12\left(x_{1}-y_{1}\right)^{2} \theta_{3}$ and $\operatorname{ker} \pi_{L}=\mathbb{R} \cdot \frac{\partial}{\partial y_{1}}$ at $\Sigma_{1}\left(\pi_{L}\right)=\left\{2 \theta_{2}+\right.$ $\left.12\left(x_{1}-y_{1}\right)^{2} \theta_{3}=0\right\}$. Since $\frac{\partial}{\partial y_{1}}\left(2 \theta_{2}+12\left(x_{1}-y_{1}\right)^{2} \theta_{3}\right)=-24\left(x_{1}-y_{1}\right) \theta_{3}$ and $\frac{\partial^{2}}{\partial y_{1}^{2}}\left(2 \theta_{2}+\right.$ $\left.12\left(x_{1}-y_{1}\right)^{2} \theta_{3}\right)=24 \theta_{3} \neq 0$, the cusp set is

$$
\Sigma_{1,1}\left(\pi_{L}\right)=\left\{2 \theta_{2}+12\left(x_{1}-y_{1}\right)^{2} \theta_{3}=x_{1}-y_{1}=0\right\}=\left\{\theta_{2}=x_{1}-y_{1}=0\right\} .
$$

Noting that $2 \theta_{2}+12\left(x_{1}-y_{1}\right)^{2} \theta_{3}$ and $x_{1}-y_{1}$ have linearly independent gradients, we see that $\pi_{L}$ has a cusp singularity.

Similarly, the projection to the right is

$\pi_{R}\left(x, y_{1}, \theta_{2}, \theta_{3}\right)=\left(y_{1}, x_{2}-\left(x_{1}-y_{1}\right)^{2}, x_{3}-\left(x_{1}-y_{1}\right)^{4},-2\left(x_{1}-y_{1}\right) \theta_{2}-4\left(x_{1}-y_{1}\right)^{3} \theta_{3}, \theta_{2}, \theta_{3}\right)$, so that $\operatorname{det} d \pi_{R}=-2 \theta_{2}-12\left(x_{1}-y_{1}\right)^{2} \theta_{3}$, and $\operatorname{ker} \pi_{R}=\frac{\partial}{\partial x_{1}}$ at $\Sigma_{1}\left(\pi_{R}\right)=\left\{2 \theta_{2}+\right.$ $\left.12\left(x_{1}-y_{1}\right)^{2} \theta_{3}=0\right\}$. One also has $\frac{\partial}{\partial x_{1}}\left(2 \theta_{2}+12\left(x_{1}-y_{1}\right)^{2} \theta_{3}\right)=24\left(x_{1}-y_{1}\right) \theta_{3}$ and $\frac{\partial^{2}}{\partial x_{1}^{2}}\left(2 \theta_{2}+12\left(x_{1}-y_{1}\right)^{2} \theta_{3}\right)=24 \theta_{3} \neq 0$, so that $\Sigma_{1,1}\left(\pi_{R}\right)=\left\{\theta_{2}=x_{1}-y_{1}=0\right\}$, and thus $\pi_{R}$ also has a cusp singularity.

Note that $\Sigma_{1}\left(\pi_{R}\right)=\Sigma_{1}\left(\pi_{L}\right)$ and $\Sigma_{1,1}\left(\pi_{R}\right)=\Sigma_{1,1}\left(\pi_{L}\right)=: \Sigma_{1,1}$. The first of these is true for any canonical relation [19], but the second is a very strong condition; indeed, for general two-sided cusps, there is no relationship between $\Sigma_{1,1}\left(\pi_{L}\right)$ and $\Sigma_{1,1}\left(\pi_{R}\right)$. Furthermore, for the images of these sets, one has $\pi_{L}\left(\Sigma_{1}\right)=\left\{\xi_{2}^{3}=-(27 / 8) \xi_{1}^{2} \xi_{3}\right\}$ and $\pi_{R}\left(\Sigma_{1}\right)=\left\{\eta_{2}^{3}=-(27 / 8) \eta_{1}^{2} \eta_{3}\right\}$, with $\pi_{L}\left(\Sigma_{1,1}\right)=\left\{\xi_{1}=\xi_{2}=0\right\}, \quad \pi_{R}\left(\Sigma_{1,1}\right)=$ $\left\{\eta_{1}=\eta_{2}=0\right\}$, resp., their cuspidal edges. $\pi_{L}\left(\Sigma_{1,1}\right)$ and $\pi_{R}\left(\Sigma_{1,1}\right)$ are codimension two coisotropic, since the Poisson bracket $\left\{\xi_{1}, \xi_{2}\right\}=0$, and nonradial, since $\xi \cdot \partial_{\xi} \notin$ $\left(T \pi_{L}\left(\Sigma_{1,1}\right)\right)^{\omega}=\operatorname{span}\left\{\partial_{x_{1}}, \partial_{x_{2}}\right\}$, and similarly for $\pi_{R}$.

Consider next the composition of canonical relations,

$$
C_{0}^{t} \circ C_{0}=\left\{(x, \xi ; y, \eta): \exists(z, \zeta) \text { s.t. }(x, \xi ; z, \zeta) \in C_{0}^{t} \text { and }(z, \zeta ; y, \eta) \in C_{0}\right\} \text {. }
$$


For a triple $(x, \xi ; z, \zeta ; y, \eta)$ contributing to $C_{0}^{t} \circ C_{0}$, the following equations hold:

$$
\begin{gathered}
z_{2}-x_{2}=\left(z_{1}-x_{1}\right)^{2}, \quad z_{3}-x_{3}=\left(z_{1}-x_{1}\right)^{4}, \\
\xi_{1}=\zeta_{1}=-2\left(z_{1}-x_{1}\right) \zeta_{2}-4\left(z_{1}-x_{1}\right)^{3} \zeta_{3}, \xi_{2}=\zeta_{2}, \xi_{3}=\zeta_{3}, \\
z_{2}-y_{2}=\left(z_{1}-y_{1}\right)^{2}, \quad z_{3}-y_{3}=\left(z_{1}-y_{1}\right)^{4}, \\
\eta_{1}=\zeta_{1}=-2\left(z_{1}-y_{1}\right) \zeta_{2}-4\left(z_{1}-y_{1}\right)^{3} \zeta_{3}, \eta_{2}=\zeta_{2}, \quad \eta_{3}=\zeta_{3} .
\end{gathered}
$$

Using $2\left(z_{1}-x_{1}\right) \xi_{2}+4\left(z_{1}-x_{1}\right)^{3} \xi_{3}=2\left(z_{1}-y_{1}\right) \xi_{2}+4\left(z_{1}-y_{1}\right)^{3} \xi_{3}$, after simplification one obtains:

$$
\begin{gathered}
\left(y_{1}-x_{1}\right) \xi_{2}+2\left[\left(z_{1}-x_{1}\right)^{3}-\left(z_{1}-y_{1}\right)^{3}\right] \xi_{3}=0, \\
\left(y_{1}-x_{1}\right)\left[\xi_{2}+2\left(3 z_{1}^{2}-3 z_{1}\left(x_{1}+y_{1}\right)+x_{1}^{2}+y_{1}^{2}+x_{1} y_{1}\right) \xi_{3}\right]=0
\end{gathered}
$$

It follows that the contribution to $C_{0}^{t} \circ C_{0}$ from $\left\{y_{1}-x_{1}=0\right\}$ is contained in $\Delta$, and that from $\left\{y_{1}-x_{1} \neq 0\right\}$ is contained in $\widetilde{C}_{0}$, where

$$
\begin{gathered}
\widetilde{C}_{0}=\left\{\left(x_{1}, x_{2}, x_{3},-2\left(z_{1}-x_{1}\right) \theta_{2}-4\left(z_{1}-x_{1}\right)^{3} \theta_{3}, \theta_{2}, \theta_{3} ;\right.\right. \\
\left.y_{1}, y_{2}, y_{3},-2\left(z_{1}-x_{1}\right) \theta_{2}-4\left(z_{1}-x_{1}\right)^{3} \theta_{3}, \theta_{2}, \theta_{3}\right): \\
x \in \mathbb{R}^{3}, y_{1}, z_{1} \in \mathbb{R}, \theta_{3} \in \mathbb{R} \backslash 0, y_{2}=x_{2}+\left(y_{1}-x_{1}\right)\left(2 z_{1}-x_{1}-y_{1}\right), \\
y_{3}=x_{3}+\left(y_{1}-x_{1}\right)\left(2 z_{1}-x_{1}-y_{1}\right)\left(\left(z_{1}-x_{1}\right)^{2}+\left(z_{1}-y_{1}\right)^{2}\right), \\
\left.\theta_{2}=-2\left(3 z_{1}^{2}-3 z_{1}\left(x_{1}+y_{1}\right)+x_{1}^{2}+y_{1}^{2}+x_{1} y_{1}\right) \theta_{3}\right\}
\end{gathered}
$$

Notice that $\xi_{1}=\eta_{1}=4 \theta_{3}\left(z_{1}-x_{1}\right)\left(z_{1}-y_{1}\right)\left(2 z_{1}-x_{1}-y_{1}\right)$ and that $\widetilde{C}_{0}$ intersects $\Delta$ in codimension one, at $\left\{x_{1}-y_{1}=0\right\}$.

The parametrization of $\widetilde{C}_{0}$ in (3) is a map

$$
\Upsilon: \mathbb{R}_{x_{1}, x_{2}, x_{3}, y_{1}, z_{1}}^{5} \times\left(\mathbb{R}_{\theta_{3}} \backslash 0\right) \longrightarrow\left(T^{*} \mathbb{R}^{3} \backslash 0\right) \times\left(T^{*} \mathbb{R}^{3} \backslash 0\right)
$$

One easily sees that $\Upsilon$ is singular at $\widetilde{\Sigma}:=\Sigma_{1}(\Upsilon)=\left\{x_{1}-y_{1}=x_{1}-z_{1}=0\right\}$, where ker $d \Upsilon=\mathbb{R} \cdot \frac{\partial}{\partial z_{1}}$, which is $\not \subset T \Sigma_{1}$. Thus, as discussed below, $\widetilde{C}_{0}$ is an open umbrella, exhibiting the simplest kind of singularity of a Lagrangian manifold.

2.3. Open umbrellas. For maps between manifolds of the same dimension, a fold is a singularity of the type $S_{1,0}$ and a cusp is a singularity of type $S_{1,1,0}[33,27,10]$. From larger dimensional spaces to smaller ones, $S_{1,0}$ maps are submersions with folds, while in the opposite direction they are referred to variously as cross caps or WhitneyCayley umbrellas [10,9]. In the lowest possible dimensions, if $g: R^{2} \rightarrow R^{3}$ has a cross cap singularity then, in suitable local coordinates, $(u, v, w)=g(x, y)=\left(x^{2}, y, x y\right)$, and its image is the algebraic surface $\left\{w^{2}=u v^{2}\right\}$, the Whitney-Cayley umbrella. This is the simplest type of nonimmersed surface singularity in three dimensions. It is an immersion away from the origin; it is actually an embedding on $\{y \neq 0\}$; and along $\{y=0\}$, it is folded and hence $2-1$.

Now, by adding one dimension to the range space, one may simultaneously both unfold the closed umbrella, making the parametrization 1-1 away from the origin, and make it a Lagrangian manifold with singularity in $\mathbb{R}^{4}$. The open (or unfolded) umbrella is the map $U: R^{2} \rightarrow R^{4}, U(x, y)=\left(x^{2}, y, x y, \frac{2}{3} x^{3}\right)$ (and its image). We have $U^{*} \omega=0$, where $\omega$ is the symplectic form on $R^{4} \simeq T^{*} \mathbb{R}^{2}$. Hence, the unfolded umbrella is a Lagrangian inclusion, i.e., a variety which is a smoothly immersed Lagrangian 
manifold away from its singular points [9]. To find a similar conic model, one needs to look in $T^{*} R^{3} \backslash 0$; for an unknown function $f(t, s)$, consider

$$
\Lambda_{1}=\left\{\left(s^{2}, t, f(t, s) ; s t \theta, \frac{2}{3} t^{3} \theta, \theta\right) \in T^{*} \mathbb{R}^{3} \backslash 0: \theta \neq 0\right\} .
$$

Then, $\Lambda_{1}$ is a Lagrangian if $f$ is chosen so that $\left.d \xi \wedge d x\right|_{\Lambda_{1}}=0$. Since $d \xi \wedge d x=$ $d(s t \theta) \wedge d\left(t^{2}\right)+d\left(\frac{2}{3} t^{3} \theta\right) \wedge d s+d \theta \wedge d f=d \theta \wedge\left(2 s t^{2} d t+\frac{2}{3} t^{3} d s\right)+d \theta \wedge d f$, this holds if $d f=-2 s t^{2} d t-\frac{2}{3} t^{3} d s=-d\left(\frac{2}{3} t^{3} s\right) ;$ using $f=-\frac{2}{3} t^{3} s$ yields the conic Lagrangian,

$$
\Lambda_{1}=\left\{\left(t^{2}, s,-\frac{2}{3} t^{3} s ; s t \theta, \frac{2}{3} t^{3} \theta, \theta\right): t, s \in \mathbb{R}, \theta \in \mathbb{R} \backslash 0\right\},
$$

exhibiting an open umbrella singularity along $\left\{\left(0,0,0 ; 0,0, \xi_{3}\right): \xi_{3} \neq 0\right\}$.

Observe that $\Lambda_{1}$ may be parametrized by a degenerate phase function. Letting $\varphi\left(x, \theta_{1}, \theta_{2}, \tau, \eta\right)=\left(x_{1}-\left(\frac{\tau}{\eta}\right)^{2}\right) \theta_{1}+\left(x_{3}+\frac{2}{3}\left(\frac{\tau}{\eta}\right)^{3} x_{2}\right) \theta_{2}$ on a conic nhood of $(0 ; 0,0,0,1)$, one has

$$
\begin{array}{cl}
d_{\theta_{1}} \varphi=x_{1}-\left(\frac{\tau}{\eta}\right)^{2}, & d_{\theta_{2}} \varphi=x_{3}+\frac{2}{3}\left(\frac{\tau}{\eta}\right)^{3} x_{2}, \\
d_{\tau} \varphi=\frac{\tau}{\eta}\left(-2 \frac{\theta_{1}}{\eta}+2 \frac{\tau}{\eta} \frac{\theta_{2}}{\eta} x_{2}\right), & d_{\eta} \varphi=-\left(\frac{\tau}{\eta}\right)^{2}\left(-2 \frac{\theta_{1}}{\eta}+2 \frac{\tau}{\eta} \frac{\theta_{2}}{\eta} x_{2}\right),
\end{array}
$$

so that $\varphi$ is degenerate, with both $d_{\tau} \varphi$ and $d_{\eta} \varphi$ having normal crossings: $d_{\tau} \varphi=0$ iff $\tau=0$ or $-2 \frac{\theta_{1}}{\eta}+2 \frac{\tau}{\eta} \frac{\theta_{2}}{\eta} x_{2}=0$, and $d_{\eta} \varphi=0$ on same two sets. For the second case, solving for $\theta_{1}=\frac{\tau}{\eta} x_{2} \theta_{2}$ we see that $\varphi$ parametrizes $\Lambda_{1}$, while for the first case, $\varphi$ also parametrizes $\Lambda_{0}:=N^{*}\left\{x_{1}=x_{3}=0\right\} . \Lambda_{0}$ and $\Lambda_{1}$ intersect cleanly in codimension one, except at the singular set of $\Lambda_{1}$, which is contained in the intersection. $\varphi$ simultaneously parametrizes $\Lambda_{0} \cup \Lambda_{1}$, although it is not a multiphase function in the sense of [26], used to parametrize a pair of cleanly intersecting smooth Lagrangians. The phase functions encountered in the composition calculations below have similar features.

For the general notion of an open umbrella, let $(M, \omega)$ be a smooth symplectic manifold of dimension $2 n$.

Definition 2.3. $S \subset M$ is an open umbrella if it is the image of a map $\psi: R^{n} \rightarrow M$ such that $d \psi$ drops rank by one simply at $\Sigma_{1}$, of codim two; $\left.\operatorname{ker}(d \psi)\right|_{\Sigma_{1}} \not \subset T \Sigma_{1}$; and Range $\left(d \psi_{p}\right)$ is Lagrangian for $p \in R^{n} \backslash \Sigma$.

The class of open umbrellas is structurally stable under smooth perturbations in the class of Lagrangian inclusions [9]. As checked above, the parametrization $\Upsilon$ in (3) satisfies the conditions in the definition, so that $\widetilde{C}_{0}$ is an open umbrella.

2.4. Composition for the model flat two sided cusp. We now want to show that the composition $A^{*} A$ for Fourier integral operators, such as $R_{0}$, associated with the model flat two-sided cusp $C_{0}$ in (2) results in operators with wave front relation in the union of the diagonal $\Delta$ and the open umbrella $\widetilde{C}_{0}$ from (3). Let $A \in I^{m}\left(\mathbb{R}^{3}, \mathbb{R}^{3} ; C_{0}\right)$, so that $A f(x)=\int e^{i \phi(x, y, \theta)} a(x, y, \theta) f(y) d \theta d y$ with $\left.\phi(x, y, \theta)=\left(x_{2}-y_{2}-\left(x_{1}-y_{1}\right)^{2}\right)\right) \theta_{2}$ $+\left(x_{3}-y_{3}-\left(x_{1}-y_{1}\right)^{4}\right) \theta_{3}$ and $a \in S_{1,0}^{m+\frac{1}{2}}$, the standard Hörmander class of symbols. We consider the normal operator formed by the composition,

$$
A^{*} A f(x)=\int e^{i(\phi(z, y, \eta)-\phi(z, x, \theta))} a(z, y, \eta) \bar{a}(z, x, \theta) f(y) d y d z d \theta d \eta .
$$


We have

$$
\begin{aligned}
\phi(z, y, \eta)-\phi(z, x, \theta)= & \left.\left(z_{2}-y_{2}-\left(z_{1}-y_{1}\right)^{2}\right)\right) \eta_{2}+\left(z_{3}-y_{3}-\left(z_{1}-y_{1}\right)^{4}\right) \eta_{3} \\
& \left.-\left(z_{2}-x_{2}-\left(z_{1}-x_{1}\right)^{2}\right)\right) \theta_{2}-\left(z_{3}-x_{3}-\left(z_{1}-x_{1}\right)^{4}\right) \theta_{3} .
\end{aligned}
$$

After a stationary phase in $\left(z_{2}, \eta_{2}\right)$ and $\left(z_{3}, \eta_{3}\right)$ in the integral for $K_{A^{*} A}$, we obtain:

$$
\begin{aligned}
\phi-\phi= & \left(x_{2}-y_{2}+\left(z_{1}-x_{1}\right)^{2}-\left(z_{1}-y_{1}\right)^{2}\right) \theta_{2} \\
& +\left(x_{3}-y_{3}+\left(z_{1}-x_{1}\right)^{4}-\left(z_{1}-y_{1}\right)^{4}\right) \theta_{3} \\
= & \left(x_{2}-y_{2}\right) \theta_{2}+\left(x_{3}-y_{3}\right) \theta_{3} \\
& +\left(y_{1}-x_{1}\right)\left[\left(2 z_{1}-x_{1}-y_{1}\right) \theta_{2}+\left(2 z_{1}-y_{1}-x_{1}\right)\left(\left(z_{1}-x_{1}\right)^{2}+\left(z_{1}-y_{1}\right)^{2}\right) \theta_{3}\right]
\end{aligned}
$$

Let $z_{1}=\frac{\zeta}{\theta_{3}}$, and then consider the change of variable,

$$
\theta_{1}=-\left(2 \frac{\zeta}{\theta_{3}}-x_{1}-y_{1}\right) \theta_{2}-\left(2 \frac{\zeta}{\theta_{3}}-y_{1}-x_{1}\right)\left(\left(\frac{\zeta}{\theta_{3}}-x_{1}\right)^{2}+\left(\frac{\zeta}{\theta_{3}}-y_{1}\right)^{2}\right) \theta_{3},
$$

for which

$$
\begin{aligned}
\frac{\partial \theta_{1}}{\partial \zeta} & \left.=-2 \frac{\theta_{2}}{\theta_{3}}-2\left(\left(\frac{\zeta}{\theta_{3}}-x_{1}\right)^{2}+\left(\frac{\zeta}{\theta_{3}}-y_{1}\right)^{2}\right)\right)-2\left(2 \frac{\zeta}{\theta_{3}}-x_{1}-y_{1}\right)^{2}, \\
\frac{\partial^{2} \theta_{1}}{\partial \zeta^{2}} & =-\frac{12}{\theta_{3}}\left(2 \frac{\zeta}{\theta_{3}}-x_{1}-y_{1}\right), \text { and } \quad \frac{\partial^{3} \theta_{1}}{\partial \zeta^{3}}=-\frac{24}{\theta_{3}} .
\end{aligned}
$$

When $\frac{\partial^{2} \theta_{1}}{\partial \zeta^{2}}=0$ then $2 \frac{\zeta}{\theta_{3}}=x_{1}+y_{1}, \theta_{1}=0$ and $\theta_{2}=-\frac{\left(x_{1}-y_{1}\right)^{2}}{2} \theta_{3}$. Let $T\left(t_{1}, t_{2}\right)=$ $\left(t_{1} t_{2}+\frac{1}{2} t_{1}^{3}, t_{2}\right)$ be a normal form for a two-dimensional map with a cusp singularity. To understand the push forward $T_{*} u$ of a distribution $u$ under the map $T$, note that the kernel of $T_{*}$ is

$$
K_{T_{*}}(z, t)=\delta(z-T(t))=\int e^{i\left[\left(z_{1}-t_{1} t_{2}-\frac{1}{2} t_{1}^{3}\right) \sigma_{1}+\left(z_{2}-t_{2}\right) \sigma_{2}\right]} 1(\sigma) d \sigma,
$$

so that

$$
T_{*} u(z)=\int e^{i\left[z_{1} \sigma_{1}+z_{2} \sigma_{2}\right]}\left[\int e^{-i\left[t_{2} \sigma_{2}+\left(t_{1} t_{2}+\frac{1}{2} t_{1}^{3}\right) \sigma_{1}\right]} u(t) d t\right] d \sigma .
$$

Applying this to $A^{*} A$, letting $z_{1}=\frac{\theta_{1}}{\theta_{3}}$ and $z_{2}=\frac{\theta_{2}}{\theta_{3}}+\frac{1}{2}\left(x_{1}-y_{1}\right)^{2}$, the phase function of $A^{*} A$ becomes

$$
\begin{aligned}
\widetilde{\phi}(x, y, \theta, \sigma)=\left(x_{1}-y_{1}\right) \theta_{1} & +\left(x_{2}-y_{2}\right) \theta_{2}+\left(x_{3}-y_{3}\right) \theta_{3} \\
& +\left(\frac{\theta_{1}}{\theta_{3}}-t_{1} t_{2}-\frac{1}{2} t_{1}^{3}\right) \sigma_{1}+\left(\frac{\theta_{2}}{\theta_{3}}+\frac{1}{2}\left(x_{1}-y_{1}\right)^{2}-t_{2}\right) \sigma_{2}
\end{aligned}
$$

and the amplitude becomes: $a \times 1(\sigma)$. Next, we perform stationary phase, first in $t_{2}, \sigma_{2}$ and then in $\theta_{1}, \sigma_{1}$. We have $d_{t_{2}} \widetilde{\phi}=-t_{1} \sigma_{1}-\sigma_{2}$ and $d_{\sigma_{2}} \widetilde{\phi}=\frac{\theta_{2}}{\theta_{3}}+\frac{1}{2}\left(x_{1}-y_{1}\right)^{2}-t_{2}$, with the Hessian $=1$; the new phase function, $\widetilde{\phi}=(x-y) \cdot \theta+\left(\frac{\theta_{1}}{\theta_{3}}-\frac{1}{2} t_{1}^{3}-t_{1}\left[\frac{\theta_{2}}{\theta_{3}}+\right.\right.$ $\left.\left.\frac{1}{2}\left(x_{1}-y_{1}\right)^{2}\right]\right) \sigma_{1}$, then has

$$
d_{\theta_{1}} \widetilde{\phi}=x_{1}-y_{1}+\frac{\sigma_{1}}{\theta_{3}}, \quad d_{\sigma_{1}} \widetilde{\phi}=\frac{\theta_{1}}{\theta_{3}}-\frac{1}{2} t_{1}^{3}-t_{1}\left(\frac{\theta_{2}}{\theta_{3}}+\frac{1}{2}\left(x_{1}-y_{1}\right)^{2}\right),
$$

wih Hessian $\frac{1}{\theta_{3}^{2}}$. Finally, we introduce the variable $\tau=t_{1} \theta_{3}$ and obtain

$$
\widetilde{\phi}=\left(x_{2}-y_{2}+\frac{\tau}{\theta_{3}}\left(x_{1}-y_{1}\right)\right) \theta_{2}+\left(x_{3}-y_{3}+\frac{1}{2}\left(x_{1}-y_{1}\right)\left(\frac{\tau}{\theta_{3}}\right)^{3}+\frac{1}{2} \frac{\tau}{\theta_{3}}\left(x_{1}-y_{1}\right)^{3}\right) \theta_{3},
$$


with respect to which the Schwartz kernel has the representation

$$
K_{A^{*} A}(x, y)=\int e^{i \widetilde{\phi}} b d \theta_{2} d \theta_{3} d \tau, \quad b \in S^{2 m} .
$$

The wave front relation satisfies

$$
\begin{aligned}
& W F\left(K_{A^{*} A}\right)^{\prime} \subset\left\{\left(x_{1}, x_{2}, x_{3}, \frac{\tau \theta_{2}}{\theta_{3}}+\frac{1}{2} \frac{\tau^{3}}{\theta_{3}^{2}}+\frac{3}{2}\left(x_{1}-y_{1}\right)^{2} \tau, \theta_{2}, \theta_{3} ;\right.\right. \\
& \left.y_{1}, y_{2}, y_{3}, \frac{\tau \theta_{2}}{\theta_{3}}+\frac{1}{2} \frac{\tau^{3}}{\theta_{3}^{2}}+\frac{3}{2}\left(x_{1}-y_{1}\right)^{2} \tau, \theta_{2}, \theta_{3}\right): \\
& d_{\theta_{2}} \widetilde{\phi}=x_{2}-y_{2}+\frac{\tau}{\theta_{3}}\left(x_{1}-y_{1}\right)=0 \\
& d_{\theta_{3}} \widetilde{\phi}=x_{3}-y_{3}-\frac{\theta_{2} \tau}{\theta_{3}^{2}}\left(x_{1}-y_{1}\right)-\frac{\tau^{3}}{\theta_{3}^{3}}\left(x_{1}-y_{1}\right)=0, \\
& \left.d_{\tau} \widetilde{\phi}=\frac{\theta_{2}}{\theta_{3}}\left(x_{1}-y_{1}\right)+\frac{3}{2} \frac{\tau^{2}}{\theta_{3}^{2}}\left(x_{1}-y_{1}\right)+\frac{1}{2}\left(x_{1}-y_{1}\right)^{3}=0\right\} .
\end{aligned}
$$

From the last relation, we see that the phase function is degenerate, with the critical set in the phase variables having a normal crossing, being the union of two transverse surfaces, corresponding to $x_{1}-y_{1}=0$ or $\frac{\theta_{2}}{\theta_{3}}+\frac{3}{2} \frac{\tau^{2}}{\theta_{3}^{2}}+\frac{1}{2}\left(x_{1}-y_{1}\right)^{2}=0$. The points where $x_{1}=y_{1}$ give rise to the diagonal $\Delta$, but with the parametrization actually being a cusp:

$$
(x, \tau, \theta) \longrightarrow\left(x, \frac{\tau \theta_{2}}{\theta_{3}}+\frac{1}{2} \frac{\tau^{3}}{\theta_{3}^{2}}, \theta_{2}, \theta_{3} ; \quad x, \frac{\tau \theta_{2}}{\theta_{3}}+\frac{1}{2} \frac{\tau^{3}}{\theta_{3}^{2}}, \theta_{2}, \theta_{3}\right) .
$$

On the other hand, the points where $\frac{\theta_{2}}{\theta_{3}}+\frac{3}{2} \frac{\tau^{2}}{\theta_{3}^{2}}+\frac{1}{2}\left(x_{1}-y_{1}\right)^{2}=0$ contribute to $W F\left(A^{*} A\right)$ the set, parametrized by $x \in \mathbb{R}^{3}, y_{1}, \tau \in \mathbb{R}, \theta_{3} \neq 0$,

$$
\begin{aligned}
\widetilde{C}_{0}=\left\{(x, \xi ; y, \eta): y_{2}\right. & =x_{2}+\frac{\tau}{\theta_{3}}\left(x_{1}-y_{1}\right), \quad \xi_{3}=\eta_{3}=\theta_{3}, \\
y_{3} & =x_{3}+\frac{1}{2} \frac{\tau}{\theta_{3}}\left(x_{1}-y_{1}\right)^{3}+\frac{1}{2} \frac{\tau^{3}}{\theta_{3}^{3}}\left(x_{1}-y_{1}\right), \\
\xi_{1} & =\eta_{1}=-\frac{\tau^{3}}{\theta_{3}^{2}}+\left(x_{1}-y_{1}\right)^{2} \tau, \\
\xi_{2} & \left.=\eta_{2}=-\frac{1}{2}\left(x_{1}-y_{1}\right)^{2} \theta_{3}-\frac{3}{2} \frac{\tau^{2}}{\theta_{3}}\right\},
\end{aligned}
$$

which is the image of $\rho\left(x_{1}, x_{2}, x_{3}, y_{1}, \theta_{3}, \tau\right)=(x, \xi ; y, \eta)$ with $y_{2}, y_{3}, \eta_{1}, \eta_{2}, \eta_{3}, \xi_{1}, \xi_{2}, \xi_{3}$ given by the above relations. $\rho$ satisfies the conditions of Def. 2.3: $d \rho$ drops rank simply by one at $\Sigma=\left\{x_{1}-y_{1}=\tau=0\right\}$ and ker $d \rho=\mathbb{R} \cdot \frac{\partial}{\partial \tau} \nsubseteq T \Sigma$. Hence $\widetilde{C}_{0}$ is an open umbrella.

Next, we compute the principal symbols on $\Delta$ and $\widetilde{C}_{0}$ away from their intersection. We have $\operatorname{Crit}_{\widetilde{\phi}}=\operatorname{Crit}_{\Delta} \cup \operatorname{Crit}_{\widetilde{C}_{0}}=\left\{\left(x, y, \tau, \theta_{2}, \theta_{3}\right) \mid d_{\tau} \widetilde{\phi}=0, d_{\theta_{2}} \widetilde{\phi}=0, d_{\theta_{3}} \widetilde{\phi}=0\right\}$. Using Hörmander's formula [19], $\sigma=a\left(E_{\widetilde{\phi}}\right)^{\frac{1}{2}}$ where $E_{\widetilde{\phi}}=\left|\frac{D\left(\lambda_{i}, \frac{\partial \widetilde{\phi}}{\partial \theta}\right)}{D(x, \theta)}\right|^{-1}$ and $\lambda_{i}$ are local coordinates on $C_{r i t_{\tilde{\phi}}}$. On $\operatorname{Crit}_{\Delta}$, local coordinates are $\left(x, \tau, \theta_{2}, \theta_{3}\right)$ and $E_{\widetilde{\phi}}=$ $\left|\frac{D\left(x, \tau, \theta_{2}, \theta_{3}, \frac{\partial \widetilde{\phi}}{\partial \tau}, \frac{\partial \widetilde{\phi}}{\partial \theta_{2}}, \frac{\partial \widetilde{\phi}}{\partial \theta_{3}}\right)}{D\left(x, \tau, \theta_{2}, \theta_{3}, y_{1}, y_{2}, y_{3}\right)}\right|^{-1}=\left(\frac{\theta_{2}}{\theta_{3}}+\frac{3}{2} \frac{\tau^{2}}{\theta_{3}^{2}}\right)^{-1}$, while, on $C r i \tau_{\widetilde{C}_{0}}$, local coordinates are 
$\left(x, y_{1}, \tau, \theta_{3}\right)$ and $E_{\widetilde{\phi}}=\left|\frac{D\left(x, y_{1}, \tau, \theta_{3}, \frac{\partial \widetilde{\phi}}{\partial \tau}, \frac{\partial \widetilde{\phi}}{\partial \theta_{2}}, \frac{\partial \widetilde{\phi}}{\partial \theta_{3}}\right)}{D\left(x, y_{1}, \tau, \theta_{2}, \theta_{3}, y_{2}, y_{3}, \theta_{2}\right)}\right|^{-1}=\left(\frac{x_{1}-y_{1}}{\theta_{3}}\right)^{-1}$. Thus, both principal symbols are singular as one approaches $\Delta \cap \widetilde{C}_{0}$, behaving as $\delta^{-\frac{1}{2}}$, where $\delta$ is the distance on either $\Delta$ or $\widetilde{C}_{0}$ to the intersection.

2.5. Flat two-sided cusps. Motivated by the previous example, we now define the class of canonical relations for which we will establish a composition calculus in Sec. 5 .

Definition 2.4. If $X$ and $Y$ are manifolds of dimension $n \geq 3$, then a canonical relation $C \subset\left(T^{*} X \backslash 0\right) \times\left(T^{*} Y \backslash 0\right)$ is a flat two-sided cusp if

(i) both $\pi_{L}: C \longrightarrow T^{*} X$ and $\pi_{R}: C \longrightarrow T^{*} Y$ have at most cusp singularities;

(ii) the left- and right-cusp points are equal: $\Sigma_{1,1}\left(\pi_{L}\right)=\Sigma_{1,1}\left(\pi_{R}\right)=: \Sigma_{1,1}$; and

(iii) $\pi_{L}\left(\Sigma_{1,1}\right) \subset T^{*} X$ and $\pi_{R}\left(\Sigma_{1,1}\right) \subset T^{*} Y$ are coisotropic and nonradial, cf. Def. 2.1.

Here, as usual, at most cusp means either cusps or folds, which are unavoidable in the neighborhood of a cusp.

Other examples of generalized Radon transforms giving flat two-sided cusps, verified as for the example above, are translates of curves in $\mathbb{R}^{4}$ with $\operatorname{span}\left\{\dot{\gamma}, \ddot{\gamma}, \dddot{\gamma}, \gamma^{(4)}\right\}=$ $\mathbb{R}^{4}$, and translates of Menn's surface [1] in $\mathbb{R}^{3}$, or any other surface whose Gauss map has a cusp.

Remarks. 1. If (i) and (ii) in Def. 2.4 are satisfied, the structure of $\left.\omega_{T^{*} X}\right|_{T \pi_{L}\left(\Sigma_{1,1}\right)}$ is the same as $\left.\omega_{T^{*} Y}\right|_{T \pi_{R}\left(\Sigma_{1,1}\right)}$, since both are equivalent to the restriction to $\Sigma_{1,1} \subset C$ of the degenerate symplectic form $\omega_{C}:=\pi_{L}^{*} \omega_{T^{*} X}=\pi_{R}^{*} \omega_{T^{*} Y}$. Thus, $\pi_{L}\left(\Sigma_{1,1}\right)$ is coisotropic (involutive) iff $\pi_{R}\left(\Sigma_{1,1}\right)$ is, and one can see that this holds iff the twodimensional $\operatorname{ker}\left(\left.\omega_{C}\right|_{\Sigma_{1}}\right)$ is simply tangent to $\Sigma_{1}$ at $\Sigma_{1,1}$. This is in contrast to the situation when the image of the cusp set is symplectic (strongly noninvolutive), in which case $\omega_{C}$ is a folded symplectic form and $\operatorname{ker}\left(\left.\omega_{C}\right|_{\Sigma_{1}}\right) \pitchfork \Sigma_{1}$ everywhere [22].

2. Condition (i) is stable under small perturbations, since cusps are structurally stable [10]. However, given (i), condition (ii) is unstable and atypical. To see this concretely, consider (possibly) nontranslationally invariant families of curves $\left\{\gamma_{x}\right\}_{x \in \mathbb{R}^{4}}$, using the framework of [4] as analyzed in terms of FIOs with cusps in [12]. For vector fields $X, Y, Z, W$ on $\mathbb{R}^{4}$, with $X \neq 0$, let $\gamma_{x}(t)=\exp _{x}\left(t X+t^{2} Y+t^{3} Z+t^{4} W\right)$. The associated generalized Radon transform is in $I^{-\frac{1}{2}}\left(\mathbb{R}^{4}, \mathbb{R}^{4} ; C\right)$, with $C$ a canonical relation for which, by $\left[12\right.$, Prop. 6.1], $\pi_{L}$ (resp., $\pi_{R}$ ) is at most a cusp if

$$
X, Y, Z \pm \frac{1}{6}[X, Y], W \pm \frac{1}{4}[X, Z]+\frac{1}{24}[X,[X, Y]] \text { are linearly independent. }
$$

From the calculations in [12], one sees that a necessary condition for $\Sigma_{1,1}\left(\pi_{L}\right)=$ $\Sigma_{1,1}\left(\pi_{R}\right)$ is that $\left(Z+\frac{1}{6}[X, Y]\right)-\left(Z-\frac{1}{6}[X, Y]\right) \in \operatorname{span}\{X, Y\}$, i.e., $\operatorname{span}\{X, Y\}$ is an integrable distribution of 2-planes, which is generically not the case. Nevertheless, as seen in the next section, flat two-sided cusps arise naturally in a quite different setting without translation invariance or integrability.

3. It is natural to ask whether curves of the form $\gamma_{m n}(t)=\left(t, t^{m}, t^{n}\right)$ for $m, n \in \mathbb{N}$, $m<n$, generate other interesting classes of canonical relations. However, the critical set $\Sigma_{1}$ is smooth iff $m=2$, and for $m=2$ (as is well known), $n=3$ gives rise to a two-sided fold; $n=4$ to the model of interest here; and $n \geq 5$ to a canonical relation whose projections do not belong to any stable singularity classes. In particular, these more degenerate cases do not seem to be of direct interest in the seismology problem 
that we now discuss, where the caustics of primary interest, those that are structurally stable, e.g., folds, cusps, swallowtails, etc., give rise to projections of the same type.

\section{Linearized seismic inversion with cusp caustics}

We consider linearized seismic inversion for the single source data set, under the assumption that the background sound speed has caustics of at most cusp type, i.e., either folds or cusps. We show that the linearized forward scattering operator $F$ is a Fourier integral operator associated with a flat two-sided cusp, $\widetilde{C}$. We only briefly describe this problem, referring to $[29,30,28,6,7]$ for more motivation and details.

Acoustic waves are generated at the surface of the earth, scatter off heterogeneities in the subsurface and return to the surface, where measurements of the pressure field are used to reconstruct an image of the subsurface. The model for the scattered waves is given by the acoustic wave equation,

$$
\frac{1}{c^{2}(x)} \frac{\partial^{2} p}{\partial t^{2}}(x, t)-\triangle p(x, t)=\delta(x-s) \delta(t), \quad p(x, t)=0, \quad t<0,
$$

where $x \in Y=\left\{x \in R^{3}, x_{3} \geq 0\right\}$, representing the Earth, $p(x, t)$ is the pressure field resulting from a pulse at the source $s$ at time $t=0$, and $c(x)$ is the unknown sound speed field.

To make this nonlinear inverse problem tractable, one considers a linearized operator $F$ which maps singular perturbations of a smooth background sound speed in the subsurface, assumed known, to perturbations of the resulting pressure field at the surface. Thus, the linearization consists in assuming $c$ to be of the form $c=c_{0}+\delta c$ and the resulting $p=p_{0}+\delta p$, where $c_{0}$ is a smooth known background field. The formal linearization of $(5)$ is

(6) $\square_{c_{0}}(\delta p):=\frac{1}{c_{0}^{2}(x)} \frac{\partial^{2} \delta p}{\partial t^{2}}(x, t)-\triangle \delta p(x, t)=\frac{2}{c_{0}^{3}} \frac{\partial^{2} p_{0}}{\partial t^{2}} \cdot \delta c(x), \quad \delta p=0, \quad t<0$,

where $p_{0}$, the Green's function for $\square_{c_{0}}$. The linearized scattering operator is $F$ : $\left.\delta c \rightarrow \delta p\right|_{\partial Y \times(0, T)}$. Under mild technical assumptions, $F$ is an FIO associated with a canonical relation $C \subset\left(T^{*} X \backslash 0\right) \times\left(T^{*} Y \backslash 0\right)$, where $X=\partial Y \times(0, T)$ is the data space $[31,20,30]$. The goal is then to left-invert $F$; standard techniques suggest studying left invertibility of the normal operator, $N=F^{*} F$.

Let $H(x, \xi)=\frac{1}{2}\left(c_{0}(x)^{-2}-|\xi|^{2}\right)$ be the Hamiltonian associated to $c_{0}$, and $\Lambda_{s}$ the image of $T_{s}^{*} \mathbb{R}^{3} \backslash 0$ under the bicharacteristic flow associated to $H$, which is a Lagrangian submanifold of $T^{*} \mathbb{R}^{3} \backslash 0$. A caustic is a singularity of the spatial projection $\pi: \Lambda_{s} \rightarrow Y$. It is known that the caustics exhibited for generic soundspeeds $c_{0}$ are the same as generic Lagrangian singularities, i.e., folds, cusps, swallowtails, etc. [17].

If $c_{0}$ is such that only fold caustics occur, it was shown by Nolan [28] that $F \in$ $I^{1}(X, Y ; C)$ with $C$ a two-sided fold. Furthermore, the Schwartz kernel of the operator $F^{*} F$ belongs to a class of distributions associated to two cleanly intersecting Lagrangians in $\left(T^{*} Y \backslash 0\right) \times\left(T^{*} Y \backslash 0\right)$, with the corresponding canonical relations being the diagonal $\Delta$ and another folding canonical relation $[28,6]$.

The next caustics to consider are those of cusp type, meaning that the only singularities of the spatial projection $\pi: \Lambda_{s} \rightarrow Y$ are folds or cusps; a cusp caustic was already present in the 2D example of [29] exhibiting loss of regularity for $F$. The goal of the remainder of this section is to prove Thm. 1.1 concerning the structure of $C$. 
Proof. To analyze the geometry of the canonical relation $C$ in the presence of cusp caustics, we make use of the description of $\Lambda_{s}$ in [28]. It can be parametrized by $t_{i n c}$, the time travelled by the incident ray, and the takeoff direction $\left(p_{1}, p_{2}, p_{3}\right) \in \mathbb{S}^{2}$. We can change these coordinates to $\left(x_{1}, x_{2}, p_{3}\right)$, so that $x_{3}=f\left(x_{1}, x_{2}, p_{3}\right),\left(p_{1}, p_{2}\right)=$ $\left(g_{1}\left(x_{1}, x_{2}, p_{3}\right), g_{2}\left(x_{1}, x_{2}, p_{3}\right)\right) . \Lambda_{s}$ is then the graph of a function $\nabla G\left(x_{1}, x_{2}, p_{3}\right)$, so that $\frac{\partial G}{\partial x_{1}}=g_{1}, \frac{\partial G}{\partial x_{2}}=g_{2}, \frac{\partial G}{\partial p_{3}}=f$; note that $\frac{\partial g_{1}}{\partial p_{3}}=\frac{\partial f}{\partial x_{1}}, \frac{\partial g_{2}}{\partial p_{3}}=\frac{\partial f}{\partial x_{2}}, \frac{\partial g_{2}}{\partial x_{1}}=\frac{\partial g_{1}}{\partial x_{2}}$. In these coordinates, $\pi\left(x_{1}, x_{2}, p_{3}\right)=\left(x_{1}, x_{2}, f\left(x_{1}, x_{2}, p_{3}\right)\right)$, det $d \pi=\frac{\partial f}{\partial p_{3}}$ and cusp caustics occur when

(7) $\frac{\partial f}{\partial p_{3}}=\frac{\partial^{2} f}{\partial p_{3}^{2}}=0, \quad \frac{\partial^{3} f}{\partial p_{3}^{3}} \neq 0$, and $\left\{\nabla \frac{\partial f}{\partial p_{3}}, \nabla \frac{\partial^{2} f}{\partial p_{3}^{2}}\right\}$ are linearly independent.

Next, we parametrize the canonical relation $C$ of $F$ in terms of $x_{1}, x_{2}$ and $p_{3}$; $\left(\alpha_{1}, \alpha_{2}, \sqrt{1-|\alpha|^{2}}\right)$, the take off direction of the reflected ray, writing $\alpha=\left(\alpha_{1}, \alpha_{2}\right)$; and $\tau$, the variable dual to time.

$$
\begin{aligned}
C=\{ & \left(r_{1}(\cdot), r_{2}(\cdot), t_{i n c}(\cdot)+t_{r e f}(\cdot), \rho_{1}(\cdot), \rho_{2}(\cdot), \tau ; x_{1}, x_{2}, f(\cdot),\right. \\
& \left.\left.-\tau\left(c_{0}^{-1}(\cdot) \alpha_{1}+g_{1}(\cdot)\right),-\tau\left(c_{0}^{-1}(\cdot) \alpha_{2}+g_{2}(\cdot)\right),-\tau\left(c_{0}^{-1}(\cdot) \sqrt{1-|\alpha|^{2}}+p_{3}\right)\right)\right\},
\end{aligned}
$$

where

$$
\begin{aligned}
f(\cdot)= & f\left(x_{1}, x_{2}, p_{3}\right) ; \quad r_{j}(\cdot)=r_{j}\left(x_{1}, x_{2}, f\left(x_{1}, x_{2}, p_{3}\right), \alpha\right), j=1,2 ; \\
t_{\text {inc }}(\cdot)= & t_{\text {inc }}\left(x_{1}, x_{2}, p_{3}\right) ; \quad t_{r e f}(\cdot)=t_{r e f}\left(x_{1}, x_{2}, f\left(x_{1}, x_{2}, p_{3}\right), \alpha\right) ; \\
\rho_{j}(\cdot)= & \rho_{j}\left(x_{1}, x_{2}, f\left(x_{1}, x_{2}, p_{3}\right), \alpha\right), j=1,2 ; \quad g_{j}(\cdot)=g_{j}\left(x_{1}, x_{2}, p_{3}\right), j=1,2 ; \\
& \quad \text { and } c_{0}^{-1}(\cdot)=c_{0}^{-1}\left(x_{1}, x_{2}, f(\cdot)\right) .
\end{aligned}
$$

We have $\pi_{R}\left(x_{1}, x_{2}, p_{3}, \alpha_{1}, \alpha_{2}, \tau\right)=\left(x_{1}, x_{2}, f(\cdot),-\tau\left(c_{0}^{-1}(\cdot) \alpha_{1}+g_{1}(\cdot)\right),-\tau\left(c_{0}^{-1}(\cdot) \alpha_{2}+\right.\right.$ $\left.g_{2}(\cdot)\right),-\tau\left(c_{0}^{-1}(\cdot) \sqrt{1-|\alpha|^{2}}+p_{3}\right)$ and thus

$$
d \pi_{R}=\left[\begin{array}{cccccc}
1 & 0 & 0 & 0 & 0 & 0 \\
0 & 1 & 0 & 0 & 0 & 0 \\
\frac{\partial f}{\partial x_{1}} & \frac{\partial f}{\partial x_{2}} & \frac{\partial f}{\partial p_{3}} & 0 & 0 & 0 \\
A & B & C & -\tau c_{0}^{-1} & 0 & -c_{0}^{-1}(\cdot) \alpha_{1}-g_{1}(\cdot) \\
D & E & F & 0 & -\tau c_{0}^{-1} & -c_{0}^{-1}(\cdot) \alpha_{2}-g_{2}(\cdot) \\
G & H & I & \frac{\tau c_{0}^{-1} \alpha_{1}}{\sqrt{1-|\alpha|^{2}}} & \frac{\tau c_{0}^{-1} \alpha_{2}}{\sqrt{1-|\alpha|^{2}}} & -c_{0}^{-1}(\cdot) \sqrt{1-|\alpha|^{2}}-p_{3}
\end{array}\right]
$$

where $A=\frac{\partial\left(-\tau\left(c_{0}^{-1}(\cdot) \alpha_{1}+g_{1}(\cdot)\right)\right)}{\partial x_{1}} ; B=\frac{\partial\left(-\tau\left(c_{0}^{-1}(\cdot) \alpha_{1}+g_{1}(\cdot)\right)\right)}{\partial x_{2}} ; C=\frac{\partial\left(-\tau\left(c_{0}^{-1}(\cdot) \alpha_{1}+g_{1}(\cdot)\right)\right)}{\partial p_{3}}$; $D=\frac{\partial\left(-\tau\left(c_{0}^{-1}(\cdot) \alpha_{2}+g_{2}(\cdot)\right)\right)}{\partial x_{1}} ; E=\frac{\partial\left(-\tau\left(c_{0}^{-1}(\cdot) \alpha_{2}+g_{2}(\cdot)\right)\right)}{\partial x_{2}} ; F=\frac{\partial\left(-\tau\left(c_{0}^{-1}(\cdot) \alpha_{2}+g_{2}(\cdot)\right)\right)}{\partial p_{3}} ; G=$ $\frac{\partial\left(-\tau\left(c_{0}^{-1}(\cdot) \sqrt{1-|\alpha|^{2}}+p_{3}\right)\right)}{\partial x_{1}} ; H=\frac{\partial\left(-\tau\left(c_{0}^{-1}(\cdot) \sqrt{1-|\alpha|^{2}}+p_{3}\right)\right)}{\partial x_{2}} ;$ and $I=\frac{\partial\left(-\tau\left(c_{0}^{-1}(\cdot) \sqrt{1-|\alpha|^{2}}+p_{3}\right)\right)}{\partial p_{3}}$. Hence $\operatorname{det} d \pi_{R}=\frac{\partial f}{\partial p_{3}}$ and the kernel at $\Sigma_{1}=\left\{\operatorname{det} d \pi_{R}=0\right\}$ is spanned by a vector $v_{1}$ which is $\frac{\partial}{\partial p_{3}}$ plus a linear combination of $\left\{\frac{\partial}{\partial \alpha_{1}}, \frac{\partial}{\partial \alpha_{2}}, \frac{\partial}{\partial \tau}\right\}$, because the matrix

$$
\left[\begin{array}{ccc}
-\tau c_{0}^{-1} & 0 & c_{0}^{-1}(\cdot) \alpha_{1}+g_{1}(\cdot) \\
0 & -\tau c_{0}^{-1} & c_{0}^{-1}(\cdot) \alpha_{2}+g_{2}(\cdot) \\
\frac{\tau c_{0}^{-1} \alpha_{1}}{\sqrt{1-|\alpha|^{2}}} & \frac{\tau c_{0}^{-1} \alpha_{2}}{\sqrt{1-|\alpha|^{2}}} & c_{0}^{-1}(\cdot) \sqrt{1-|\alpha|^{2}}+p_{3}
\end{array}\right]
$$


is nondegenerate by the calculations of [28]. From the cusp conditions (7), one can see that $\pi_{R}$ has a cusp singularity.

Similarly, $\pi_{L}\left(x_{1}, x_{2}, \alpha_{1}, \alpha_{2}, p_{3}, \tau\right)=\left(r_{1}, r_{2}, t_{\text {inc }}+t_{r e f}, \rho_{1}, \rho_{2}, \tau\right)$ and

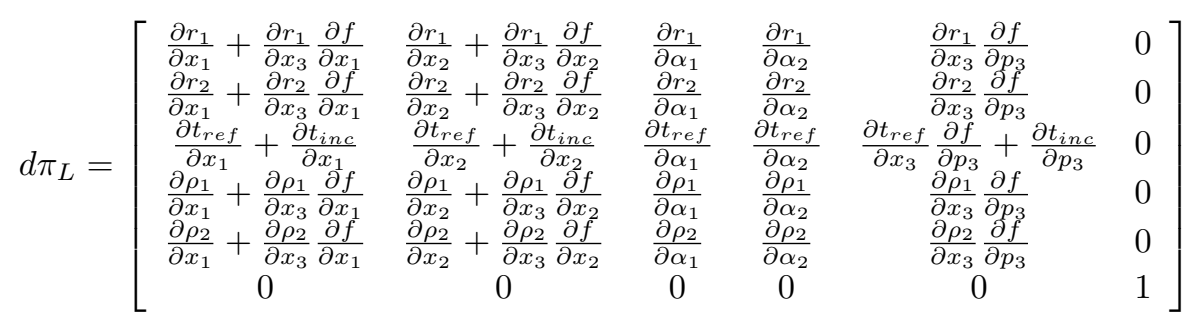

From [28] we have that $\frac{\partial t_{i n c}}{\partial p_{3}}=0, \frac{\partial r_{1}}{\partial x_{3}}=0, \frac{\partial r_{2}}{\partial x_{3}}=0, \frac{\partial \rho_{1}}{\partial x_{3}}=0, \frac{\partial \rho_{2}}{\partial x_{3}}=0, \frac{\partial t_{r e f}}{\partial x_{3}}<0$, and that the matrix

$$
\left[\begin{array}{llll}
\frac{\partial r_{1}}{\partial x_{1}} & \frac{\partial r_{1}}{\partial x_{2}} & \frac{\partial r_{1}}{\partial \alpha_{1}} & \frac{\partial r_{1}}{\partial \alpha_{2}} \\
\frac{\partial r_{2}}{\partial x_{1}} & \frac{\partial r_{2}}{\partial x_{2}} & \frac{\partial r_{2}}{\partial \alpha_{1}} & \frac{\partial r_{2}}{\partial \alpha_{2}} \\
\frac{\partial \rho_{1}}{\partial x_{1}} & \frac{\partial \rho_{1}}{\partial x_{2}} & \frac{\partial \rho_{1}}{\partial \alpha_{1}} & \frac{\partial \rho_{1}}{\partial \alpha_{2}} \\
\frac{\partial \rho_{2}}{\partial x_{1}} & \frac{\partial \rho_{2}}{\partial x_{2}} & \frac{\partial \rho_{2}}{\partial \alpha_{1}} & \frac{\partial \rho_{2}}{\partial \alpha_{2}}
\end{array}\right]
$$

is nondegenerate. Thus, $\operatorname{det} d \pi_{L}=\frac{\partial f}{\partial p_{3}}, \operatorname{ker} \pi_{L}=\frac{\partial}{\partial p_{3}}$ and as before one sees that $\pi_{L}$ has also a cusp singularity. Thus, $C$ is a two-sided cusp.

The next step in the proof of Thm. 1.1 is to show that the images of the cusp points are coisotropic. By Remark 1 in Sec. 2.5, it is enough to examine the degenerate symplectic form $\omega_{C}=\pi_{R}^{*} \omega_{T^{*} Y}$ on $C$ and its kernel, equal to $\left\{v: \omega_{C}(v, w)=0, \forall w \in\right.$ $T C\}$. We have from $(7)$ that $\Sigma_{1}=\left\{(x, \xi) ; f_{p_{3}}=0\right\}$ and $\Sigma_{1,1}=\left\{(x, \xi) ; f_{p_{3}}=f_{p_{3} p_{3}}=\right.$ $0\}$. Since $\left.\omega_{C}\right|_{\Sigma_{1}}$ drops rank by 2 and ker $d \pi_{R}=\mathbb{R} \cdot v_{1}$, one has $\operatorname{ker} \omega_{C}=\operatorname{span}\left\{v_{1}, v_{2}\right\}$, where $v_{2}$ is a vector, without $\frac{\partial}{\partial p_{3}}$ component, that we now determine. At $\Sigma_{1}$, one has

$$
\begin{aligned}
\left.\omega_{C}\right|_{\Sigma_{1}}= & \left(c_{0}^{-1} \alpha_{1}+g_{1}+\left(c_{0}^{-1} \sqrt{1-|\alpha|^{2}}+p_{3}\right) f_{x_{1}}\right)\left(d \tau \wedge d x_{1}\right) \\
+ & \left(c_{0}^{-1} \alpha_{2}+g_{2}+\left(c_{0}^{-1} \sqrt{1-|\alpha|^{2}}+p_{3}\right) f_{x_{2}}\right)\left(d \tau \wedge d x_{2}\right) \\
+ & \tau c_{0}^{-1}\left(\left(1-\frac{\alpha_{1}}{\sqrt{1-|\alpha|^{2}}} f_{x_{1}}\right)\left(d \alpha_{1} \wedge d x_{1}\right)+\left(1-\frac{\alpha_{2}}{\sqrt{1-|\alpha|^{2}}} f_{x_{2}}\right)\left(d \alpha_{2} \wedge d x_{2}\right)\right) \\
+ & \tau c_{0}^{-1} \frac{-\alpha_{1}}{\sqrt{1-|\alpha|^{2}}} f_{x_{2}}\left(d \alpha_{1} \wedge d x_{2}\right)+\tau c_{0}^{-1} \frac{-\alpha_{2}}{\sqrt{1-|\alpha|^{2}}} f_{x_{1}}\left(d \alpha_{2} \wedge d x_{1}\right) \\
+ & \tau\left(\partial_{x_{2}} c_{0}^{-1} \alpha_{1}-\partial_{x_{1}} c_{0}^{-1} \alpha_{2}+\partial_{x_{2}} c_{0}^{-1} \sqrt{1-|\alpha|^{2}} f_{x_{1}}-\partial_{x_{1}} c_{0}^{-1} \sqrt{1-|\alpha|^{2}} f_{x_{2}}\right) \\
& \times\left(d x_{2} \wedge d x_{1}\right) \\
+ & 2 \tau f_{x_{1}}\left(d p_{3} \wedge d x_{1}\right)+2 \tau f_{x_{2}}\left(d p_{3} \wedge d x_{2}\right) .
\end{aligned}
$$

We consider the $5 \times 5$ skew symmetric matrix of $\omega_{C}$ corresponding to $x_{1}, x_{2}, \alpha_{1}, \alpha_{2}, \tau$ :

$$
\left[\begin{array}{ccccc}
0 & a & \star & \star & -b \\
-a & 0 & \star & \star & -c \\
\tau c_{0}^{-1}\left(1-\frac{\alpha_{1}}{\sqrt{1-|\alpha|^{2}}} f_{x_{1}}\right) & \tau c_{0}^{-1} \frac{-\alpha_{1}}{\sqrt{1-|\alpha|^{2}}} f_{x_{2}} & 0 & 0 & 0 \\
\tau c_{0}^{-1} \frac{-\alpha_{2}}{\sqrt{1-|\alpha|^{2}}} f_{x_{1}} & \tau c_{0}^{-1}\left(1-\frac{\alpha_{2}}{\sqrt{1-|\alpha|^{2}}} f_{x_{2}}\right) & 0 & 0 & 0 \\
b & c & 0 & 0 & 0
\end{array}\right]
$$


where $a$ is the coefficient of $d x_{1} \wedge d x_{2}, b$ is the coefficient of $d \tau \wedge d x_{1}$ and $c$ is the coefficient of $d \tau \wedge d x_{2} . v$ is a combination of $\frac{\partial}{\partial x_{1}}, \frac{\partial}{\partial x_{2}}, \frac{\partial}{\partial \alpha_{1}}, \frac{\partial}{\partial \alpha_{2}}, \frac{\partial}{\partial \tau}$; note that

$$
\left|\begin{array}{cc}
1-\frac{\alpha_{1}}{\sqrt{1-|\alpha|^{2}}} f_{x_{1}} & \frac{-\alpha_{1}}{\sqrt{1-|\alpha|^{2}}} f_{x_{2}} \\
\frac{-\alpha_{2}}{\sqrt{1-|\alpha|^{2}}} f_{x_{1}} & 1-\frac{\alpha_{2}}{\sqrt{1-|\alpha|^{2}}} f_{x_{2}}
\end{array}\right|=1+\nabla \sqrt{1-|\alpha|^{2}} \cdot \nabla_{x_{1}, x_{2}} f \neq 0,
$$

which means that $v_{2}$ does not contain any $\frac{\partial}{\partial x_{1}}, \frac{\partial}{\partial x_{2}}$ terms, but only $\frac{\partial}{\partial \alpha_{1}}, \frac{\partial}{\partial \alpha_{2}}, \frac{\partial}{\partial \tau}$. This shows that ker $\omega_{C}$ is simply tangent to $\Sigma_{1}$ at $\Sigma_{1,1}$, and thus $\pi_{R}\left(\Sigma_{1,1}\right)$ is coisotropic; by Remark 1, so is $\pi_{L}\left(\Sigma_{1,1}\right)$. This finishes the proof of Thm 1.1.

\section{Weak normal forms for flat two-sided cusps}

Next, we show that any flat two-sided cusp can be prepared, by application of suitable canonical transformations on the left and right, so as to be parametrized by a phase function similar to that for $R_{0}$ in (1). Recall that one can conjugate any two-sided fold to a single normal form $[22,23]$, but, as in $[16,7]$, we will need to work with merely approximate normal forms. For simplicity, we restrict ourselves to the three dimensional setting of interest for the seismic problem. Assume that $\operatorname{dim}(X)=\operatorname{dim}(Y)=3$ and suppose $C \subset T^{*} X \times T^{*} Y$ is a flat two-sided cusp. Let $c^{0} \in \Sigma_{1,1}$ and set $\left(x^{0}, \xi^{0}\right):=\pi_{L}\left(c^{0}\right),\left(y^{0}, \eta^{0}\right):=\pi_{R}\left(c^{0}\right)$. The first step in finding a weak normal from is to show that there exist local canonical coordinates on $T^{*} X$ and $T^{*} Y$ allowing for a phase function parametrizing $C$ using the same variables as for the model $C_{0}$ in Sec. 2 .

Proposition 4.1. There exist local canonical coordinates $(x, \xi)$ on $T^{*} X$ near $\left(x^{0}, \xi^{0}\right)$ and $(y, \eta)$ on $T^{*} Y$ near $\left(y^{0}, \eta^{0}\right)$ such that, $\left(x_{1}, x^{\prime}, y_{1}, \eta^{\prime}\right):=\left(x_{1}, x_{2}, x_{3}, y_{1}, \eta_{2}, \eta_{3}\right)$ form local coordinates on $C$ near $c^{0}$. Thus, there exists a generating function $S\left(x, y_{1}, \theta^{\prime}\right)$ so that $\phi\left(x, y, \theta^{\prime}\right)=S\left(x, y_{1}, \theta^{\prime}\right)-y^{\prime} \cdot \theta^{\prime}$ parametrizes $C$ near $c^{0}$.

Proof. Since $d \pi_{L}$ and $d \pi_{R}$ drop rank by 1 at $c^{0}$, there exist (see [19]) symplectic decompositions $T_{\left(x^{0}, \xi^{0}\right)}\left(T^{*} X\right)=V_{1} \oplus V^{\prime}, T_{\left(y^{0}, \eta^{0}\right)}\left(T^{*} Y\right)=W_{1} \oplus W^{\prime}$, with $\operatorname{dim}\left(V_{1}\right)=$ $\operatorname{dim}\left(W_{1}\right)=2, \operatorname{dim}\left(V^{\prime}\right)=\operatorname{dim}\left(W^{\prime}\right)=4$, and

$$
T_{c^{0}} C=G r(\chi) \oplus\left(\Lambda_{L} \times(0)\right) \oplus\left((0) \times \Lambda_{R}\right),
$$

where $\Lambda_{L} \subset V_{1}, \Lambda_{R} \subset W_{1}$ are Lagrangian, i.e., one-dimensional, subspaces and $\chi \in$ $S p\left(V^{\prime}, W^{\prime}\right)$. Thus, in suitable linear symplectic coordinates, $\Lambda_{L}=\left\{\xi_{1}=0\right\}, \Lambda_{R}=$ $\left\{\eta_{1}=0\right\}$ in $V_{1} \simeq W_{1} \simeq T^{*} \mathbb{R}$, and $\chi=I \in S p\left(T^{*} \mathbb{R}^{2}, T^{*} \mathbb{R}^{2}\right)$, so that $\left(x_{1}, y_{1}, x^{\prime}, \eta^{\prime}\right)$ are coordinates on $T_{c^{0}} C$. By a standard application of the homogeneous Darboux theorem [19, 22], there are local canonical coordinates $(x, \xi, y, \eta)$ on $T^{*} X \times T^{*} Y$ such that $c^{0}=\left(0, e_{3}^{*} ; 0, e_{3}^{*}\right)$ and $\left(x, y_{1}, \eta^{\prime}\right)$ form local coordinates on $C$.

We further prepare $C$ by noting that if $v_{R}, v_{L}$ are kernel vector fields for $\pi_{R}, \pi_{L}$, i.e., nonzero vector fields along $\Sigma_{1}$ generating $\operatorname{ker}\left(d \pi_{R}\right), \operatorname{ker}\left(d \pi_{L}\right)$, resp., then $v_{L}$ is a linear combination of $\partial_{y_{1}}, \partial \eta_{2}, \partial \eta_{3}$. Thus, $d \pi_{R}\left(v_{L}\right)$ is a vector field along $\pi_{R}\left(\Sigma_{1,1}\right)$, nontangent to $\Sigma_{1,1}$ since $\pi_{R}$ is a cusp. Using homogeneous Darboux, one can assume that $v_{L}=\partial_{y_{1}}$ at $\Sigma_{1,1}$, and (using also the nonradiality of $\pi_{R}\left(\Sigma_{1,1}\right)$ ), that $\pi_{R}\left(\Sigma_{1,1}\right)=$ $\left\{\eta_{1}=\eta_{2}=0\right\}$ and $\operatorname{span}\left\{T \pi_{R}\left(\Sigma_{1,1}\right), d \pi_{R}\left(v_{L}\right)\right\}=d \pi_{R}(T C)=\left\{d \eta_{1}=0\right\}$ along $\Sigma_{1,1}$. Working similarly on the left, one can assume $v_{R}=\partial_{x_{1}}, \pi_{L}\left(\Sigma_{1,1}\right)=\left\{\xi_{1}=\xi_{2}=0\right\}$ and $d \pi_{L}(T C)=\left\{d \xi_{1}=0\right\}$ along $\Sigma_{1,1}$. 
Furthermore, near $c^{0}$ one can assume that (i) $\Sigma_{1}=\left\{\eta_{2}=f\left(x, y_{1}, \eta_{3}\right)\right\}$, and (ii) $\Sigma_{1,1}=\left\{\eta_{2}=f\left(x, y_{1}, \eta_{3}\right), y_{1}=g\left(x, \eta_{3}\right)\right\}$ for functions $f, g$, homogeneous of degrees 1,0 , resp., and with $\partial_{x_{1}} g \neq 0$. To see (i), let $\widetilde{f}\left(x, y_{1}, \eta^{\prime}\right)$ be a defining function for $\Sigma_{1}$, homogeneous of degree 1 ; by the implicit function theorem it suffices to show that $d_{\eta_{2}} \widetilde{f}\left(c^{0}\right) \neq 0$. Euler's identity implies that $\eta^{\prime} \cdot d_{\eta^{\prime}} \widetilde{f}=0$ at $\Sigma_{1}$; since $\eta_{2}=$ $0, \eta_{3} \neq 0$ at $c^{0}$, this yields $d_{\eta_{3}} \tilde{f}=0$. Since $\pi_{L}, \pi_{R}$ are cusps, the kernel vector fields $v_{L}, v_{R} \in T \Sigma_{1}$ at $\Sigma_{1,1}$, thus at $c^{0}$, and hence $d_{x_{1}} \widetilde{f}\left(c^{0}\right)=d_{y_{1}} \widetilde{f}\left(c^{0}\right)=0$. If $d_{\eta_{2}} \widetilde{f}=0$, the only nonzero components of $d \widetilde{f}\left(c^{0}\right)$ would be $d_{x^{\prime}} \widetilde{f}$, and this contradicts the fact that $d \pi_{L}\left(T \Sigma_{1}\right)=d \pi_{L}\left(T \Sigma_{1,1}\right)=T \pi_{L}\left(\Sigma_{1,1}\right)=\left\{d \xi_{1}=d \xi_{2}=0\right\}$. Hence, $d_{\eta_{2}} \tilde{f}\left(c^{0}\right) \neq 0$, and thus $\Sigma_{1}$ can be described as $\left\{\eta_{2}=f\left(x, y_{1}, \eta_{3}\right)\right\}$ for some $f$. Note that since $v_{R}, v_{L} \in T \Sigma_{1}$ at $\Sigma_{1,1}$, one has $\left.\partial_{x_{1}} f\right|_{\Sigma_{1,1}}=\left.\partial_{y_{1}} f\right|_{\Sigma_{1,1}}=0$. For (ii), note that since $\pi_{L}: \Sigma_{1,1} \longrightarrow \pi_{L}\left(\Sigma_{1,1}\right)$ is a diffeomorphism, and $\left(x, \xi_{3}\right)$ are coordinates on $\pi_{L}\left(\Sigma_{1,1}\right)$, it follows that $\left(\pi_{L}^{*}(x), \pi_{L}^{*}\left(\xi_{3}\right)\right)=\left(x, d_{x_{3}} S\left(x, y_{1}, \eta^{\prime}\right)\right)$ form coordinates on $\Sigma_{1,1}$. Since $T \Sigma_{1}=\operatorname{span}\left\{T \Sigma_{1,1}, v\right\}$ for both $v=v_{R}=\partial_{x_{1}}$ and $v=v_{L}=\partial_{y_{1}}$, $\left(x, y_{1}, d_{x_{3}} S\left(x, y_{1}, f, \eta_{3}\right)\right)$ are coordinates on $\Sigma_{1}$, and we can replace $d_{x_{3}} S$ by $\eta_{3}$ since $d_{x_{3} \eta_{3}}^{2} S \neq 0$. Then, $\Sigma_{1,1}$ is a hypersurface in $\Sigma_{1}$ transverse to both $\partial_{x_{1}}$ and $\partial_{y_{1}}$, and can thus be described as $\left\{\eta_{2}=f\left(x, y_{1}, \eta_{3}\right), y_{1}=g\left(x, \eta_{3}\right)\right\}$ with $\partial_{x_{1}} g \neq 0$.

Since $\left(x, y_{1}, \eta^{\prime}\right)$ form microlocal coordinates on $C$, there exists a generating function $S\left(x, y_{1}, \theta^{\prime}\right)[19]$, so that $\phi\left(x, y, \theta^{\prime}\right)=S\left(x, y_{1}, \theta^{\prime}\right)-y^{\prime} \cdot \theta^{\prime}$ parametrizes $C$ near $c^{0}$.

One can thus write

$$
C=\left\{\left(x, \partial_{x} S ; y_{1}, \partial_{\theta^{\prime}} S,-\partial_{y_{1}} S, \theta^{\prime}\right): x \in \mathbb{R}^{3}, y_{1} \in \mathbb{R}, \theta^{\prime} \in \mathbb{R}^{2} \backslash 0\right\},
$$

with $\pi_{L}\left(x, y_{1}, \theta^{\prime}\right)=\left(x, \partial_{x} S\left(x, y_{1}, \theta^{\prime}\right)\right), \pi_{R}\left(x, y_{1}, \theta^{\prime}\right)=\left(y,-\partial_{y_{1}} S\left(x, y_{1}, \theta^{\prime}\right), \theta^{\prime}\right)$ and $\Sigma_{1,1}=\left\{\theta_{2}-f=y_{1}-g=0\right\}$. Since $\pi_{L}\left(\Sigma_{1,1}\right)=\left\{\xi_{1}=\xi_{2}=0\right\}$, one has $\left.\partial_{x_{1}} S\right|_{\left\{\theta_{2}-f=y_{1}-g=0\right\}}=0$ and $\left.\partial_{x_{2}} S\right|_{\left\{\theta_{2}-f=y_{1}-g=0\right\}}=0$. From the second relation, it follows that $\left.S\right|_{\left\{\theta_{2}-f=y_{1}-g=0\right\}}$ is independent of $x_{2}$, so that $S$ is a function of just $x_{1}, x_{3}, \theta_{3}$; however, from the first relation one can express $S$ as

$$
S\left(x, y_{1}, \theta\right)=S_{0}\left(x_{3}, \theta_{3}\right)+\left(y_{1}-g\right)^{2} S_{1}\left(x, y_{1}, \theta^{\prime}\right)+\left(\theta_{2}-f\right) S_{2}\left(x, y_{1}, \theta^{\prime}\right)
$$

Similarly, $\pi_{R}\left(\Sigma_{1,1}\right)=\left\{\eta_{1}=\eta_{2}=0\right\}$ implies that $\left.\partial_{y_{1}} S\right|_{\left\{\theta_{2}-f=0=y_{1}-g\right\}}=0$, but doesn't provide any more information about $S$, cf. Remark 1. On $C$, the ideal of $\Sigma_{1,1}$ equals $\left(\theta_{2}-f\left(x, y_{1}, \theta_{3}\right), y_{1}-g\left(x, \theta_{3}\right)\right)=\left(S_{y_{1}}, \theta_{2}\right)$, so $f=\alpha S_{y_{1}}+\beta \theta_{2}$. Hence $\left.f\right|_{\Sigma_{1,1}}=0$; $\left.\partial_{\theta_{3}} f\right|_{\Sigma_{1,1}}=0$ (since $f$ is homogeneous of degree 1 ); $f=c\left(y_{1}-g\right)^{2}$ for some $c \in C^{\infty}$; and $\left.\partial_{\theta_{3}} g\right|_{\Sigma_{1,1}}=0$, since $g$ is homogeneous of degree 0 . Thus, the canonical relation must have the form,

$$
\begin{gathered}
C=\left\{\left(x_{1}, x_{2}, x_{3},-2\left(y_{1}-g\right) \partial_{x_{1}} g S_{1}+\left(y_{1}-g\right)^{2} \partial_{x_{1}} S_{1}+\left(\theta_{2}-f\right) \partial_{x_{1}} S_{2}-\partial_{x_{1}} f S_{2},\right.\right. \\
\left(y_{1}-g\right)^{2} \partial_{x_{2}} S_{1}-2\left(y_{1}-g\right) \partial_{x_{2}} g S_{1}+\left(\theta_{2}-f\right) \partial_{x_{2}} S_{2}-\partial_{x_{2}} f S_{2}, \\
\left(y_{1}-g\right)^{2} \partial_{x_{3}} S_{1}-2\left(y_{1}-g\right) \partial_{x_{3}} g S_{1}+\left(\theta_{2}-f\right) \partial_{x_{3}} S_{2}-\partial_{x_{3}} f S_{2}+\partial_{x_{3}} S_{0} \\
y_{1}, y_{2}, y_{3}, 2\left(y_{1}-g\right) S_{1}+\left(y_{1}-g\right)^{2} \partial_{y_{1}} S_{1}+\left(\theta_{2}-f\right) \partial_{y_{1}} S_{2}-\partial_{y_{1}} f S_{2}, \\
\left.-\theta_{2},-\theta_{3}\right): y_{2}=\left(y_{1}-g\right)^{2} \partial_{\theta_{2}} S_{1}+\left(\theta_{2}-f\right) \partial_{\theta_{2}} S_{2}+S_{2}, \\
\left.y_{3}=\left(y_{1}-g\right)^{2} \partial_{\theta_{3}} S_{1}-2\left(y_{1}-g\right) \partial_{\theta_{3}} g S_{1}+\left(\theta_{2}-f\right) \partial_{\theta_{3}} S_{2}-\partial_{\theta_{3}} f S_{2}+\partial_{\theta_{3}} S_{3}\right\} .
\end{gathered}
$$


From this, one sees that

$$
d \pi_{L} \equiv\left[\begin{array}{cccccc}
1 & 0 & 0 & 0 & 0 & 0 \\
0 & 1 & 0 & 0 & 0 & 0 \\
0 & 0 & 1 & 0 & 0 & 0 \\
\cdot & \cdot & \cdot & -2 \partial_{x_{1}} g S_{1}-\partial_{x_{1} y_{1}} f S_{2} & \partial_{x_{1}} S_{2} & 0 \\
\cdot & \cdot & . & -2 \partial_{x_{2}} g S_{1}-\partial_{x_{2} y_{1}} f S_{2} & \partial_{x_{2}} S_{2} & 0 \\
\cdot & . & . & . & \partial_{x_{3}} S_{2} & \partial_{x_{3} \theta_{3}}^{2} S_{0}
\end{array}\right] \bmod \left(y_{1}-g, \theta_{2}-f\right)
$$

with det $d \pi_{L}=\left[\left(-2 \partial_{x_{1}} g S_{1}-\partial_{x_{1} y_{1}} f S_{2}\right) \partial_{x_{2}} S_{2}-\left(-2 \partial_{x_{2}} g S_{1}-\partial_{x_{2} y_{1}} f S_{2}\right) \partial_{x_{1}} S_{2}\right]\left(\partial_{x_{3} \theta_{3}}^{2} S_{0}\right)$ $+\left(y_{1}-g\right) E_{1}+\left(\theta_{2}-f\right) E_{2}$ for some $E_{1}, E_{2}$. Since $\pi_{L}$ has a cusp singularity at $\Sigma_{1,1}$, the determinant must vanish simply. We have that $\left.\partial_{x_{3} \theta_{3}}^{2} S_{0}\right|_{\Sigma_{1,1}} \neq 0$ by homogeneity and $\partial_{x_{2} \theta_{2}} S=\left.\partial_{x_{2}} S_{2}\right|_{\Sigma_{1,1}} \neq 0$ since $d \pi_{L}(T C)=\left\{d \xi_{1}=0\right\}$. Since Ker $\mathrm{d} \pi_{L}=\partial_{y_{1}}$, the $y_{1}$ column in $d \pi_{L}$ must be 0 at $\Sigma_{1,1}$. We have that $\left.\left(-2 \partial_{x_{1}} g S_{1}-\partial_{x_{1} y_{1}} f S_{2}\right)\right|_{\Sigma_{1,1}}=$ $\left.\partial_{x_{1}} g\left(S_{1}+c S_{2}\right)\right|_{\Sigma_{1,1}}=0$ so $\left.\left(S_{1}+c S_{2}\right)\right|_{\Sigma_{1,1}}=0$, and $\left.\left(-2 \partial_{x_{2}} g S_{1}-\partial_{x_{2} y_{1}} f S_{2}\right)\right|_{\Sigma_{1,1}}=$ $\left.\partial_{x_{2}} g\left(S_{1}+c S_{2}\right)\right|_{\Sigma_{1,1}}=0$, and since $\partial_{x_{2}} S_{2}, \partial_{x_{1}} g \neq 0$ we can conclude that det $\left.\mathrm{d} \pi_{L}\right|_{\Sigma_{1,1}}=$ $\left.\left(S_{1}+c S_{2}\right)\right|_{\Sigma_{1,1}}$. Since $S_{0}$ is nondegenerate, by another canonical transformation on the left we may assume that $S_{0}=x_{3} \theta_{3}$. From ker $d \pi_{L}=\mathbb{R} \cdot \frac{\partial}{\partial y_{1}}$, we have $\left.\partial_{y_{1}}\left(S_{1}+c S_{2}\right)\right|_{\Sigma_{1,1}}=0$. Since $d \pi_{R}(T C)=\left\{d \eta_{1}=0\right\}$ we obtain that $\left.\partial_{y_{1}} S_{2}\right|_{\Sigma_{1,1}}=$ $\left.\partial_{\theta_{2} y_{1}} S\right|_{\Sigma_{1,1}}=0$. One also has that $\left.\partial_{y_{1}} S_{1}\right|_{\Sigma_{1,1}}=0$, since a calculation shows that this is $\left.\partial_{y_{1}}^{2} \eta_{1}\left(x, y_{1}, \theta^{\prime}\right)\right|_{\Sigma_{1,1}}$, which vanishes due to the cusp structure of $d \pi_{R}$ and the fact that $d \pi_{R}(T C)=\left\{d \eta_{1}=0\right\}$. Then one can write $S_{1}=\left(y_{1}-g\right)^{2} S_{5}+\left(\theta_{2}-f\right) S_{6}$ with $S_{5} \neq 0$, leading to

Proposition 4.2. If $C$ is a flat two-sided cusp and $c^{0} \in C$, then there exist local canonical coordinates such that $c^{0}$ has a conic nhood in $C$ parametrized by a phase function of the form

$$
\phi\left(x, y, \theta_{2}, \theta_{3}\right)=\left(S_{2}+\left(y_{1}-g\right)^{2} S_{6}\right)\left(\theta_{2}-f\right)+\left(x_{3}-y_{3}\right) \theta_{3}+\left(y_{1}-g\right)^{4} S_{5}-y_{2} \theta_{2},
$$

with $\left.\partial_{x_{2}} S_{2}\left(x, y_{1}, \theta^{\prime}\right)\right|_{\Sigma_{1,1}} \neq 0, \quad S_{5}, S_{6} \neq 0, f=f\left(x, y_{1}, \theta_{3}\right), g=g\left(x, \theta_{3}\right)$.

In studying the composition in the next section, $f, g$ introduce only algebraic complications and do not affect the final result. Thus, for simplicity we take $f=0$ and $g=x_{1}$ and will use the phase function

$$
\begin{array}{r}
\phi\left(x, y, \theta_{2}, \theta_{3}\right)=\left(S_{2}-y_{2}+\left(x_{1}-y_{1}\right)^{2} S_{4}\right) \theta_{2}+\left(x_{3}-y_{3}\right) \theta_{3}+\left(x_{1}-y_{1}\right)^{4} S_{3}, \\
\partial_{x_{2}} S_{2}\left(x, y_{1}, \theta^{\prime}\right) \neq 0, \quad S_{3}, S_{4} \neq 0 \text { on } \Sigma_{1,1},
\end{array}
$$

where $S_{5}, S_{6}$ have been relabeled as $S_{3}, S_{4}$, resp.

\section{Composition}

Now, we begin the proof of Thm. 1.2 by considering the composition $B^{*} A$ for $A \in I^{m}(X, Y ; C), B \in I^{m^{\prime}}(X, Y ; C)$. Conjugating by unitary FIOs associated with the canonical transformations used in the previous section, we may assume that $C$ is parametrized by the phase function $\phi$ from (8). We have, for $a \in S^{m+\frac{1}{2}}, b \in S^{m^{\prime}+\frac{1}{2}}$, and the phase $\widehat{\phi}=\phi\left(z, y, \eta^{\prime}\right)-\phi\left(z, x, \theta^{\prime}\right)$,

$$
B^{*} A f(x)=\int e^{i \widehat{\phi}\left(x, y, z, \eta^{\prime}, \theta^{\prime}\right)} a\left(z, y, \eta^{\prime}\right) \bar{b}\left(z, x, \theta^{\prime}\right) f(y) d y d z d \theta^{\prime} d \eta^{\prime} .
$$


We perform stationary phase in $z_{2}, \eta_{2}$ and $z_{3}, \eta_{3}$ :

$$
\begin{aligned}
& \partial_{z_{2}} \widehat{\phi}=\eta_{2} \partial_{z_{2}} S_{2}-\theta_{2} \partial_{z_{2}} S_{2}+\partial_{z_{2}} S_{3}\left(z_{1}-y_{1}\right)^{4}-\partial_{z_{2}} S_{3}\left(z_{1}-x_{1}\right)^{4} \\
& +\left(z_{1}-y_{1}\right)^{2} \eta_{2} \partial_{z_{2}} S_{4}-\left(z_{1}-x_{1}\right)^{2} \theta_{2} \partial_{z_{2}} S_{4}, \\
& \partial_{z_{3}} \widehat{\phi}=\eta_{3}-\theta_{3}+\left(z_{1}-y_{1}\right)^{4} \partial_{z_{3}} S_{3}-\left(z_{1}-x_{1}\right)^{4} \partial_{z_{3}} S_{3}+\eta_{2} \partial_{z_{3}} S_{2}-\theta_{2} \partial_{z_{3}} S_{2} \\
& +\left(z_{1}-y_{1}\right)^{2} \eta_{2} \partial_{z_{3}} S_{4}-\left(z_{1}-x_{1}\right)^{2} \theta_{2} \partial_{z_{3}} S_{4} \text {, } \\
& \text { (11) } \partial_{\eta_{2}} \widehat{\phi}=\left(z_{1}-y_{1}\right)^{4} \partial_{\eta_{2}} S_{3}+S_{2}-y_{2}+\left(z_{1}-y_{1}\right)^{2}\left[S_{4}+\eta_{2} \partial_{\eta_{2}} S_{4}\right]+\eta_{2} \partial_{\eta_{2}} S_{2} \text {, } \\
& \text { (12) } \partial_{\eta_{3}} \widehat{\phi}=z_{3}-y_{3}+\left(z_{1}-y_{1}\right)^{4} \partial_{\eta_{3}} S_{3}+\eta_{2} \partial_{\eta_{3}} S_{2}+\left(z_{1}-y_{1}\right)^{2} \eta_{2} \partial_{\eta_{3}} S_{4} \text {. }
\end{aligned}
$$

We solve for $z_{3}$ using (12) and find

$$
z_{3}=y_{3}-\left(z_{1}-y_{1}\right)^{4} \partial_{\eta_{3}} S_{3}-\eta_{2} \partial_{\eta_{3}} S_{2}-\left(z_{1}-y_{1}\right)^{2} \eta_{2} \partial_{\eta_{3}} S_{4}:=y_{3}+S_{5} .
$$

From equation (11) we can solve for $z_{2}$ since $\partial_{z_{2}} S_{2} \neq 0$, but only implicitly. Now rewrite (10) as

$$
\begin{aligned}
\eta_{3}=\theta_{3} & +\left(z_{1}-x_{1}\right)^{4} \partial_{z_{3}} S_{3}-\left(z_{1}-y_{1}\right)^{4} \partial_{z_{3}} S_{3} \\
& +\left(z_{1}-x_{1}\right)^{2} \theta_{2} \partial_{z_{3}} S_{4}-\left(z_{1}-y_{1}\right)^{2} \eta_{2} \partial_{z_{3}} S_{4}+\theta_{2} \partial_{z_{3}} S_{2}-\eta_{2} \partial_{z_{3}} S_{2},
\end{aligned}
$$

and solve for $\eta_{3}$, using the fact that $\theta_{2} \partial_{z_{3}} S_{2}-\eta_{2} \partial_{z_{3}} S_{2}$ vanishes at $\left\{x_{1}=y_{1}, \eta^{\prime}=\theta^{\prime}\right\}$, so that

$\theta_{2} \partial_{z_{3}} S_{2}-\eta_{2} \partial_{z_{3}} S_{2}=\left(x_{1}-y_{1}\right) \theta_{2} \partial_{z_{3} x_{1}}^{2} S_{2}+\left(\theta_{2}-\eta_{2}\right)\left(\partial_{z_{3}} S_{2}+\theta_{2} \partial_{z_{3} \theta_{2}}^{2} S_{2}\right)+\left(\theta_{3}-\eta_{3}\right) \theta_{2} \partial_{z_{3} \theta_{3}}^{2} S_{2}$.

The other differences in the equations (9),(12) can be handled similarly, so

$$
\begin{gathered}
\eta_{3}=\theta_{3}+\left(x_{1}-y_{1}\right) M_{1}\left(z_{1}, z_{2}, x_{1}, y_{1}, \theta^{\prime}, \eta_{2}\right)+\left(\theta_{2}-\eta_{2}\right) N_{1}\left(z_{1}, z_{2}, x_{1}, y_{1}, \theta^{\prime}, \eta_{2}\right)+ \\
\left(\theta_{3}-\eta_{3}\right) P\left(z_{1}, z_{2}, x_{1}, y_{1}, \theta^{\prime}, \eta_{2}\right)
\end{gathered}
$$

for some functions $M_{1}, N_{1}$ and $P$. Solving for $\eta_{3}-\theta_{3}$, we have

$$
\eta_{3}-\theta_{3}=\left[\left(x_{1}-y_{1}\right) M_{1}+\left(\theta_{2}-\eta_{2}\right) N_{1}\right](I+P)^{-1}=\left(x_{1}-y_{1}\right) M_{2}+\left(\theta_{2}-\eta_{2}\right) N_{2} .
$$

Also, $\eta_{2}-\theta_{2}=\left(x_{1}-y_{1}\right) M_{3}+\left(\eta_{3}-\theta_{3}\right) N_{3}$, so that $\eta_{3}-\theta_{3}=\left(x_{1}-y_{1}\right) M_{4}$ and $\eta_{2}-\theta_{2}=\left(x_{1}-y_{1}\right) N_{4}$. Hence, the phase becomes

$$
\begin{aligned}
\widehat{\phi}= & \left(x_{3}-y_{3}\right) \theta_{3}+\left(x_{2}-y_{2}\right) \theta_{2}+\left(\left(z_{1}-y_{1}\right)^{2} S_{4}\left(z_{1}, y, \theta^{\prime}\right)-\left(z_{1}-x_{1}\right)^{2} S_{4}\left(z_{1}, x_{1}, y_{2}, y_{3}, \theta^{\prime}\right)\right) \theta_{2} \\
& +\left(z_{1}-y_{1}\right)^{4} S_{3}-\left(z_{1}-x_{1}\right)^{4} S_{3}+\left(S_{2}\left(z_{1}, y, \theta^{\prime}\right)-S_{2}\left(z_{1}, x_{1}, y_{2}, y_{3}, \theta^{\prime}\right)\right) \theta_{2}+\left(x_{1}-y_{1}\right) S_{6} .
\end{aligned}
$$

We have $\left(\left(z_{1}-y_{1}\right)^{2} S_{4}\left(z_{1}, y, \theta^{\prime}\right)-\left(z_{1}-x_{1}\right)^{2} S_{4}\left(z_{1}, x_{1}, y_{2}, y_{3}, \theta^{\prime}\right)\right) \theta_{2}$

$$
\begin{aligned}
= & {\left[\left(z_{1}-y_{1}\right)^{2} S_{4}\left(z_{1}, y_{1}, \cdot\right)-\left(z_{1}-x_{1}\right)^{2} S_{4}\left(z_{1}, y_{1}, \cdot\right)\right] \theta_{2} } \\
& +\left[\left(z_{1}-x_{1}\right)^{2} S_{4}\left(z_{1}, y_{1}, \cdot\right)-\left(z_{1}-x_{1}\right)^{2} S_{4}\left(z_{1}, x_{1}, \cdot\right)\right] \theta_{2} \\
= & {\left[\left(x_{1}-y_{1}\right)\left(2 z_{1}-x_{1}-y_{1}\right) S_{4}\left(z_{1}, y_{1}, \cdot\right)+\left(z_{1}-x_{1}\right)^{2}\left(x_{1}-y_{1}\right) \partial_{y_{1}} S_{4}\right] \theta_{2}, }
\end{aligned}
$$

$\left(z_{1}-y_{1}\right)^{4} S_{3}-\left(z_{1}-x_{1}\right)^{4} S_{3}=\left(x_{1}-y_{1}\right)\left(2 z_{1}-x_{1}-y_{1}\right)\left(\left(z_{1}-y_{1}\right)^{2}+\left(z_{1}-x_{1}\right)^{2}\right) S_{3}\left(z_{1}, y_{1}, \cdot\right)+$ $\left(z_{1}-x_{1}\right)^{4}\left(x_{1}-y_{1}\right) \partial_{y_{1}} S_{3}$, and can write $\left(z_{1}-x_{1}\right)^{2}\left(x_{1}-y_{1}\right) \partial_{y_{1}} S_{4} \theta_{2}+\left(z_{1}-x_{1}\right)^{4}\left(x_{1}-\right.$ $\left.y_{1}\right) \partial_{y_{1}} S_{3}+\left(S_{2}\left(z_{1}, y, \theta^{\prime}\right)-S_{2}\left(z_{1}, x_{1}, y_{2}, y_{3}, \theta^{\prime}\right)\right) \theta_{2}+\left(x_{1}-y_{1}\right) S_{6}:=\left(x_{1}-y_{1}\right) S_{7}$. From this, it follows that $\widehat{\phi}=\left(x_{2}-y_{2}\right) \theta_{2}+\left(x_{3}-y_{3}\right) \theta_{3}+\left(x_{1}-y_{1}\right)\left[\left(2 z_{1}-x_{1}-y_{1}\right)\left(\left(z_{1}-\right.\right.\right.$ 
$\left.\left.\left.y_{1}\right)^{2}+\left(z_{1}-x_{1}\right)^{2}\right) S_{3}+\left(2 z_{1}-x_{1}-y_{1}\right) S_{4} \theta_{2}+S_{7}\left(z_{1}, y, x_{1}, \theta\right)\right]$. We make a singular change of variables similar to that in the model case in Sec. 2.4:

$$
\begin{aligned}
\theta_{1}= & \left(2 z_{1}-x_{1}-y_{1}\right)\left(\left(z_{1}-y_{1}\right)^{2}+\left(z_{1}-x_{1}\right)^{2}\right) S_{3}+\left(2 z_{1}-x_{1}-y_{1}\right) S_{4} \theta_{2}+S_{7}, \\
\frac{\partial \theta_{1}}{\partial z_{1}}= & 2 S_{4} \theta_{2}+\left(2 z_{1}-x_{1}-y_{1}\right) \theta_{2} \partial_{z_{1}} S_{4}+2 S_{3}\left(\left(z_{1}-x_{1}\right)^{2}+\left(z_{1}-y_{1}\right)^{2}\right) \\
& +2\left(2 z_{1}-x_{1}-y_{1}\right)^{2} S_{3}+\left(2 z_{1}-x_{1}-y_{1}\right)\left(\left(z_{1}-y_{1}\right)^{2}+\left(z_{1}-x_{1}\right)^{2}\right) \partial_{z_{1}} S_{3}+\partial_{z_{1}} S_{7}, \\
\frac{\partial^{2} \theta_{1}}{\partial z_{1}^{2}}= & 4 \partial_{z_{1}} S_{4} \theta_{2}+\left(2 z_{1}-x_{1}-y_{1}\right) \theta_{2} \partial_{z_{1}^{2}}^{2} S_{4}+4\left(\left(z_{1}-x_{1}\right)^{2}+\left(z_{1}-y_{1}\right)^{2}\right) \partial_{z_{1}} S_{3} \\
& +12\left(2 z_{1}-x_{1}-y_{1}\right) S_{3}+4\left(2 z_{1}-x_{1}-y_{1}\right)^{2} \partial_{z_{1}} S_{3} \\
& +\left(2 z_{1}-x_{1}-y_{1}\right)\left(\left(z_{1}-y_{1}\right)^{2}+\left(z_{1}-x_{1}\right)^{2}\right) \partial_{z_{1}}^{2} S_{3}+\partial_{z_{1}} S_{7} .
\end{aligned}
$$

Near points on $\Sigma_{1,1}, \frac{\partial^{2} \theta_{1}}{\partial z_{1}^{2}}=0$ implies $z_{1}=\frac{x_{1}+y_{1}}{2}+R\left(x_{1}, y, \theta_{2}, \theta_{3}\right)$, for some $R$ which vanishes at the base point $\left(x_{1}, y, \theta_{2}, \theta_{3}\right)=(0,0,0,0,0,1)$. Then

$$
\begin{aligned}
\frac{\partial \theta_{1}}{\partial z_{1}}= & 2 S_{4} \theta_{2}+R \partial_{z_{1}} S_{4} \theta_{2}+2 S_{3}\left(\frac{\left(x_{1}-y_{1}\right)^{2}}{2}+2 R^{2}\right) \\
& +2 R^{2} S_{3}+R\left(\frac{\left(x_{1}-y_{1}\right)^{2}}{2}+2 R^{2}\right) \partial_{z_{1}} S_{3}+\partial_{z_{1}} S_{7}=0
\end{aligned}
$$

implies that

$\theta_{2}=-\frac{\left(x_{1}-y_{1}\right)^{2}}{2} \frac{2 S_{3}+R \partial_{z_{1}} S_{3}}{2 S_{4}+R \partial_{z_{1}} S_{4}}+P_{1}\left(x_{1}, y, \theta_{3}\right)=-\frac{\left(x_{1}-y_{1}\right)^{2}}{2} N\left(x_{1}, y, \theta_{3}\right)+P_{1}\left(x_{1}, y, \theta_{3}\right)$

for some $N, P_{1}$, and $\theta_{1}=R\left(\frac{\left(x_{1}+y_{1}\right)^{2}}{2}+2 R^{2}\right) S_{3}+R S_{4} \theta_{2}+S_{7}:=P_{2}\left(x_{1}, y, \theta_{3}\right)$. Since $S_{3}, S_{4} \neq 0$ and $R=0$ at the base point, one has $N \neq 0$. Following the same analysis as in the model case, one sees that

$$
\begin{gathered}
\widehat{\phi}=\left(x_{2}-y_{2}\right) \theta_{2}+\left(x_{3}-y_{3}\right) \theta_{3}+\left(x_{1}-y_{1}\right) \theta_{1}+\left(\frac{\theta_{1}-P_{2}}{\theta_{3}}-t_{1} t_{2}-\frac{1}{2} t_{1}^{3}\right) \sigma_{1}+ \\
\left(\frac{\theta_{2}}{\theta_{3}}+\frac{\left(x_{1}-y_{1}\right)^{2}}{2} \frac{N}{\theta_{3}}-\frac{P_{1}}{\theta_{3}}-t_{2}\right) \sigma_{2} .
\end{gathered}
$$

Next, we perform a stationary phase in $t_{2}, \sigma_{2}$ :

and

$$
\partial_{t_{2}} \widehat{\phi}=-t_{1} \sigma_{1}-\sigma_{2}, \quad \partial_{\sigma_{2}} \widehat{\phi}=\frac{\theta_{2}}{\theta_{3}}+\frac{N}{2 \theta_{3}}\left(x_{1}-y_{1}\right)^{2}-\frac{P_{1}}{\theta_{3}}-t_{2}
$$

$$
\widehat{\phi}=(x-y) \theta+\left(\frac{\theta_{1}}{\theta_{3}}-\frac{P_{2}}{\theta_{3}}-t_{1}\left[\frac{\theta_{2}}{\theta_{3}}+\frac{N}{2 \theta_{3}}\left(x_{1}-y_{1}\right)^{2}-\frac{P_{1}}{\theta_{3}}\right]-\frac{1}{2} t_{1}^{3}\right) \sigma_{1}
$$

One more stationary phase in $\sigma_{1}$ and $\theta_{1}$, using

$$
\partial_{\theta_{1}} \widehat{\phi}=x_{1}-y_{1}+\frac{\sigma_{1}}{\theta_{3}}, \quad \partial_{\sigma_{1}} \widehat{\phi}=\frac{\theta_{1}}{\theta_{3}}-\frac{P_{2}}{\theta_{3}}-t_{1}\left[\frac{\theta_{2}}{\theta_{3}}+\frac{N}{2 \theta_{3}}\left(x_{1}-y_{1}\right)^{2}-\frac{P_{1}}{\theta_{3}}\right]-\frac{1}{2} t_{1}^{3},
$$

and then homogenizing $t_{1}$ by setting $\tau=t_{1} \theta_{3}$, results in the phase

$$
\text { (13) } \begin{aligned}
\widehat{\phi}=\left(x_{2}-y_{2}+\frac{\tau}{\theta_{3}}\left(x_{1}-y_{1}\right)\right) \theta_{2} & +\left(x_{3}-y_{3}+\left(x_{1}-y_{1}\right) \frac{P_{2}-\frac{\tau}{\theta_{3}} P_{1}}{\theta_{3}}\right. \\
& \left.+\left(x_{1}-y_{1}\right)^{3} \frac{\tau}{\theta_{3}^{2}} \frac{N}{2}+\left(x_{1}-y_{1}\right) \frac{1}{2}\left(\frac{\tau}{\theta_{3}}\right)^{3}\right) \theta_{3},
\end{aligned}
$$


and the Schwartz kernel is $K_{B^{*} A}(x, y)=\int e^{i \widehat{\phi}} c\left(x, y, \theta^{\prime}, \tau\right) d \theta_{2} d \theta_{3} d \tau$ with $c \in S^{m+m^{\prime}}$. For each of the variables $s=x_{1}, y_{1}, y_{2}, y_{3}$, let [[s]] denote

$$
[[s]]=\partial_{s}\left(P_{2}-\frac{\tau}{\theta_{3}} P_{1}\right)+\left(x_{1}-y_{1}\right)^{2} \frac{\tau}{\theta_{3}} \partial_{s} \frac{N}{2},\left[\left[\theta_{3}\right]\right]=\partial_{\theta_{3}}\left(P_{2}-\frac{\tau}{\theta_{3}} P_{1}+\left(x_{1}-y_{1}\right)^{2} \frac{\tau}{\theta_{3}} \frac{N}{2}+\frac{1}{2} \frac{\tau^{3}}{\theta_{3}^{2}}\right) .
$$

Then we have

$$
\begin{gathered}
W F\left(F^{*} F\right) \subset\left\{\left(x_{1}, x_{2}, x_{3}, \frac{\tau \theta_{2}}{\theta_{3}}+3\left(x_{1}-y_{1}\right)^{2} \frac{\tau N}{2 \theta_{3}}+P_{2}-\frac{\tau P_{1}}{\theta_{3}}+\frac{1}{2}\left(\frac{\tau}{\theta_{3}}\right)^{3} \theta_{3}+\left(x_{1}-y_{1}\right)\left[\left[x_{1}\right]\right], \theta^{\prime} ;\right.\right. \\
y_{1}, y_{2}, y_{3}, \frac{\tau \theta_{2}}{\theta_{3}}+3\left(x_{1}-y_{1}\right)^{2} \frac{\tau N}{2 \theta_{3}}+P_{2}-\frac{\tau P_{1}}{\theta_{3}}+\frac{1}{2}\left(\frac{\tau}{\theta_{3}}\right)^{3} \theta_{3}+\left(x_{1}-y_{1}\right)\left[\left[y_{1}\right]\right], \\
\left.\theta_{2}+\left(x_{1}-y_{1}\right)\left[\left[y_{2}\right]\right], \theta_{3}+\left(x_{1}-y_{1}\right)\left[\left[y_{3}\right]\right]\right): \\
d_{\tau} \widehat{\phi}=\left(x_{1}-y_{1}\right)\left(\frac{\theta_{2}-P_{1}}{\theta_{3}}+\frac{3}{2}\left(\frac{\tau}{\theta_{3}}\right)^{2}+\left(x_{1}-y_{1}\right)^{2} \frac{N}{2 \theta_{3}}\right)=0, \\
d_{\theta_{2}} \widehat{\phi}=x_{2}-y_{2}+\left(x_{1}-y_{1}\right) \frac{\tau}{\theta_{3}}=0, \\
\left.d_{\theta_{3}} \widehat{\phi}=x_{3}-y_{3}-\left(x_{1}-y_{1}\right) \frac{\tau \theta_{2}}{\theta_{3}^{2}}+\left(x_{1}-y_{1}\right)\left[\left[\theta_{3}\right]\right]=0\right\} .
\end{gathered}
$$

The phase function is degenerate because the equation $d_{\tau} \widehat{\phi}=0$ has a normal crossing; from $\left\{x_{1}-y_{1}=0\right\}$, the contribution to $W F\left(B^{*} A\right)$ is contained in

$$
\begin{aligned}
\Delta=\{ & \left(x, \frac{\tau \theta_{2}}{\theta_{3}}+P_{2}-\frac{\tau}{\theta_{3}} P_{1}+\frac{1}{2}\left(\frac{\tau}{\theta_{3}}\right)^{3} \theta_{3}, \theta_{2}, \theta_{3} ;\right. \\
& \left.\left.x, \frac{\tau \theta_{2}}{\theta_{3}}+P_{2}-\frac{\tau}{\theta_{3}} P_{1}+\frac{1}{2}\left(\frac{\tau}{\theta_{3}}\right)^{3} \theta_{3}, \theta_{2}, \theta_{3}\right): x \in \mathbb{R}^{3},\left(\tau, \theta^{\prime}\right) \in \mathbb{R}^{3} \backslash 0\right\},
\end{aligned}
$$

which is a cusp parametrization of $\Delta$ (as in the model case). On the other hand, if $x_{1}-y_{1} \neq 0$ then

$$
\begin{aligned}
& \theta_{2}=P_{1}-\frac{3}{2}\left(\frac{\tau}{\theta_{3}}\right)^{2} \theta_{3}-\left(x_{1}-y_{1}\right)^{2} \frac{N}{2}, \quad y_{2}=x_{2}+\left(x_{1}-y_{1}\right) \frac{\tau}{\theta_{3}}, \\
& y_{3}=x_{3}-\left(x_{1}-y_{1}\right) \frac{\tau \theta_{2}}{\theta_{3}^{2}}+\left(x_{1}-y_{1}\right)\left[\left[\theta_{3}\right]\right], \\
& \xi_{1}=P_{2}-\left(\frac{\tau}{\theta_{3}}\right)^{3} \theta_{3}+\tau N \frac{\left(x_{1}-y_{1}\right)^{2}}{\theta_{3}}+\left(x_{1}-y_{1}\right)\left[\left[x_{1}\right]\right], \\
& \eta_{1}=P_{2}-\left(\frac{\tau}{\theta_{3}}\right)^{3} \theta_{3}+\tau N \frac{\left(x_{1}-y_{1}\right)^{2}}{\theta_{3}}+\left(x_{1}-y_{1}\right)\left[\left[y_{1}\right]\right], \\
& \eta_{2}=\theta_{2}+\left(x_{1}-y_{1}\right)\left[\left[y_{2}\right]\right], \text { and } \eta_{3}=\theta_{3}+\left(x_{1}-y_{1}\right)\left[\left[y_{3}\right]\right] .
\end{aligned}
$$

Let $\widetilde{C}:=\widetilde{C}_{\widehat{\phi}}$ be the image of $\psi\left(x_{1}, x_{2}, x_{3}, y_{1}, \theta_{3}, \tau\right)=(x, \xi ; y, \eta)$ with $\xi_{2}=\theta_{2}, \xi_{3}=\theta_{3}$, and $\xi_{1}, \eta_{1}, \eta_{2}, \eta_{3}, y_{2}, y_{3}$ given above. As in the model case, $\psi$ satisfies Def. 2.3: it drops rank by one simply at $\Sigma_{1}=\left\{x_{1}-y_{1}=0=\tau\right\}$, and ker $d \pi=\mathbb{R} \cdot \frac{\partial}{\partial \tau} \nsubseteq \Phi \Sigma$, so that $\widetilde{C}$ is an open umbrella, proving the first part of Thm. 1.2.

Finally, one can compute the orders of $G$ on $\Delta \backslash \widetilde{C}$ and $\widetilde{C} \backslash \Delta$, as in the model case at the end of Sec. 2.4. Due to the normal crossing of $d_{\tau} \widehat{\phi}$, the critical set $\operatorname{Crit}_{\widehat{\phi}}=$ 
$\left\{\left(x, y, \tau, \theta^{\prime}\right): d_{\tau} \widehat{\phi}=0, d_{\theta^{\prime}} \widehat{\phi}=0\right\}$ decomposes as Crit $_{\Delta} \cup$ Crit $_{\widetilde{C}}$, and $\sigma=\rho\left(E_{\widehat{\phi}}\right)^{\frac{1}{2}}$, where $E_{\widehat{\phi}}=\left|\frac{D\left(\lambda_{i}, \frac{\partial \widehat{\phi}}{\partial \theta}\right)}{D(x, \theta)}\right|^{-1}$ and $\lambda_{i}$ are local coordinates on $C r i t_{\widehat{\phi}}$.

$\mathrm{On} \mathrm{Crit}_{\Delta}$, local coordinates are $\left(x, \tau, \theta^{\prime}\right)$ and

$$
E_{\widehat{\phi}}=\left|\frac{D\left(x, \tau, \theta^{\prime}, \frac{\partial \widehat{\phi}}{\partial \tau}, \frac{\partial \widehat{\phi}}{\partial \theta_{2}}, \frac{\partial \widehat{\phi}}{\partial \theta_{3}}\right)}{D\left(x, \tau, \theta^{\prime}, y_{1}, y_{2}, y_{3}\right)}\right|^{-1}=\left(\frac{\theta_{2}}{\theta_{3}}+\frac{3}{2} \frac{\tau^{2}}{\theta_{3}^{2}}-\frac{P_{1}}{\theta_{3}}\right)^{-1} ;
$$

hence $B^{*} A \in I^{m+m^{\prime}}(\Delta \backslash \widetilde{C})$ and the principal symbol satisfies $\sigma \sim \delta^{-\frac{1}{2}}$, where $\delta$ is the distance to $\Delta \cap \widetilde{C}$. On the other hand, $\left(x, y_{1}, \tau, \theta_{3}\right)$ are local coordinates on Crit $_{C_{\widehat{\phi}}}$,

$$
\begin{aligned}
E_{\widehat{\phi}} & =\left|\frac{D\left(x, y_{1}, \tau, \theta_{3}, \frac{\partial \widehat{\phi}}{\partial \tau}, \frac{\partial \widehat{\phi}}{\partial \theta_{2}}, \frac{\partial \widehat{\phi}}{\partial \theta_{3}}\right)}{D\left(x, y_{1}, \tau, \theta_{2}, \theta_{3}, y_{2}, y_{3}, \theta_{2}\right)}\right|^{-1} \\
& =\left(\frac{x_{1}-y_{1}}{\theta_{3}}+\frac{\left(x_{1}-y_{1}\right)^{2}}{\theta_{3}}\left[\partial_{y_{3} \theta_{3}} P_{2}-\frac{\tau}{\theta_{3}} \partial_{y_{3} \theta_{3}} P_{1}+\left(x_{1}-y_{1}\right)^{2} \frac{\tau}{2 \theta_{3}} \partial_{\left.y_{3} \theta_{3} N\right]}\right)^{-1},\right.
\end{aligned}
$$

which is $\sim\left(x_{1}-y_{1} / \theta_{3}\right)^{-1}$, so that $B^{*} A \in I^{m+m^{\prime}}(\widetilde{C} \backslash \Delta)$ and again $\sigma \sim \delta^{-\frac{1}{2}}$. The equality of the orders on $\Delta$ and $\widetilde{C}$ away from $\Delta \cap \widetilde{C}$ is consistent with the composition result in $[28,6]$ for two-sided folds. This gives a precise description of the nonremovable artifact in the linearized seismic inversion problem for the single source geometry in the presence of cusp caustics.

\section{Acknowledgements}

We would like to thank Cliff Nolan for patiently explaining some of the calculations in [28], and the referee for several suggestions. The second author was supported by NSF grants DMS-0551894 and -0853892.

\section{References}

[1] T. Banchoff, T. Gaffney and C. McCrory, Cusps of Gauss mappings, Research Notes in Math., 55, Pitman, Boston, 1982.

[2] G. Beylkin, Imaging of discontinuities in the inverse problem by inversion of a generalized Radon transform, Jour. Math. Phys. 28 (1985), 99-108.

[3] A. Comech and S. Cuccagna, Integral operators with two-sided cusp singularities, Int. Math. Res. Not. (2000), 1225-1242.

[4] M. Christ, A. Nagel, E. Stein and S. Wainger, Singular and maximal Radon transforms: analysis and geometry, Ann. of Math. (2) 150 (1999), 489-577.

[5] J.J. Duistermaat and V. Guillemin, The spectrum of positive elliptic operators and periodic bicharacteristics, Invent. Math. 29 (1975), 39-79.

[6] R. Felea, Composition calculus of Fourier integral operators with fold and blowdown singularities, Comm. P.D.E. 30 (2005), 1717-1740.

[7] R. Felea and A. Greenleaf, An FIO calculus for marine seismic imaging: folds and crosscaps, Comm. P.D.E. 33 (2008), 45-77.

[8] R. Felea, A. Greenleaf and M. Pramanik, An FIO calculus for marine seismic imaging, II: Sobolev estimates, http://arxiv.org/abs/0903.1780; Math. Annalen (2010), to appear.

[9] A. Givental, Lagrangian imbeddings of surfaces and unfolded Whitney umbrella. (English) Func. Anal. Appl. 20(3) (1986), 197-203

[10] M. Golubitsky and V. Guillemin, Stable mappings and their singularities, Springer-Verlag, New York, 1973.

[11] A. Greenleaf and A. Seeger, Fourier integral operators with fold singularities, J. Reine Angew. Math. 455 (1994), 35-56. 
$[12] \_$, Fourier integral operators with cusp singularities, Amer. Jour. Math. 120 (1998), 1077-1119.

[13] _ , Oscillatory integral operators with low-order degeneracies, Duke Math. Jour. 112 (2002), 397-420.

[14] _ Oscillatory and Fourier integral operators with degenerate canonical relations, in Proc. 6th Int. Conf. on Harmonic Analysis and Partial Diff. Eqns. (El Escorial, 2000), Publ. Mat. 2002, Vol. Extra, 93-141.

[15] A. Greenleaf and G. Uhlmann, Estimates for singular Radon transforms and pseudodifferential operators with singular symbols, Jour. Func. Anal. 89 (1990), 220-232.

[16] _ , Compositions of some singular Fourier integral operators and estimates for restricted X-ray transforms, Ann. Inst. Fourier (Grenoble) 40(2) (1990), 443-466; II, Duke Math. Jour. 64 (1991), 415-444.

[17] J. Guckenheimer, Caustics and non-degenerate Hamiltonians, Topology 13 (1974), 127-133.

[18] V. Guillemin and G. Uhlmann, Oscillatory integrals with singular symbols, Duke Math. Jour. 48 (1981), 251-267.

[19] L. Hörmander, The Analysis of Linear Partial Differential Operators, III, Grundlehren math. Wiss. 274, Springer Verlag, Berlin, 1985

[20] A. ten Kroode, D. Smit and A. Verdel, A microlocal analysis of migration, Wave Motion 28 (1998), 149-172.

[21] F. Marhuenda, Microlocal analysis of some isospectral deformations, Trans. Amer. Math. Soc. 343 (1994), 245-275.

[22] R. Melrose, Equivalence of glancing hypersurfaces. II, Math. Annalen 255 (1981), 159-198.

[23] R. Melrose and M. Taylor, Near peak scattering and the corrected Kirchhoff approximation for a convex obstacle, Adv. in Math. 55(3) (1985), 242-315.

[24] R. Melrose and G. Uhlmann, Lagrangian intersection and the Cauchy problem, Comm. Pure Appl. Math. 32(4) (1979), 483-519.

[25] _ Microlocal structure of involutive conical refraction, Duke Math. J. 46 (1979), $571-582$

[26] G. Mendoza, Symbol calculus associated with intersecting Lagrangians, Comm. P.D.E. 7 (1982), $1035-1116$.

[27] B. Morin, Formes canoniques des singularites d'une application differentiable, C.R. Acad. Sc. Paris, 260 (1965) 5662-5665 and 6503-6506.

[28] C. Nolan, Scattering in the presence of fold caustics, SIAM J. Appl. Math. 61 (2000), 659-672.

[29] C. Nolan and W. Symes, Anomalous reflections near a caustic, Wave Motion 25 (1997), 1-14.

[30] , Global solutions of a linearized inverse problem for the acoustic wave equation, Comm. P.D.E. 22 (1997), 919-952.

[31] Rakesh, A linearized inverse problem for the wave equation, Comm. P.D.E. 13 (1988), 573-601.

[32] A. Weinstein, On Maslov's quantization condition, in: Chazarain, J., ed. Fourier Integral Operators and Partial Differential Equations, Springer-Verlag, New York, 1975.

[33] H. Whitney, The general type of singularity of a set of $2 n-1$ smooth functions of $n$ variables, Duke Math. Jour. 10 (1943), 161-172.

School of Mathematical Sciences, Rochester Institute of Technology, Rochester, Ny 14623

E-mail address: rxfsma@rit.edu

Department of Mathematics, University of Rochester, Rochester, NY 14627

E-mail address: allan@math.rochester.edu 